\title{
St. Lucia: Statistical Appendix
}

This Statistical Appendix for St. Lucia was prepared by a staff team of the International Monetary Fund as background documentation for the periodic consultation with the member country. It is based on the information available at the time it was completed on April 15, 2004. The views expressed in this document are those of the staff team and do not necessarily reflect the views of the government of St. Lucia or the Executive Board of the IMF.

The policy of publication of staff reports and other documents by the IMF allows for the deletion of market-sensitive information.

To assist the IMF in evaluating the publication policy, reader comments are invited and may be sent by e-mail to publicationpolicy@imf.org.

Copies of this report are available to the public from

International Monetary Fund $\bullet$ Publication Services

700 19th Street, N.W. • Washington, D.C. 20431

Telephone: (202) 6237430 • Telefax: (202) 6237201

E-mail: publications@imf.org • Internet: http://www.imf.org

Price: $\$ 15.00$ a copy

\section{International Monetary Fund Washington, D.C.}





\title{
INTERNATIONAL MONETARY FUND
}

\author{
ST. LUCIA \\ Statistical Appendix \\ Prepared by Eric Verreydt, Claudio Visconti and Tobias Rasmussen (all WHD), and
} Philippe Karam (INS)

Approved by Westerm Hemisphere Department

April 15, 2004

Contents

Page

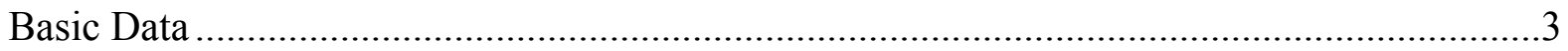

Statistical Appendix-Tables

1. Selected Economic and Financial Indicators ………....................................................

2. GDP by Economic Activity at Constant Factor Cost ......................................................6

3. GDP by Economic Activity at Current Factor Cost .......................................................

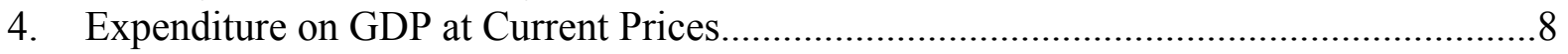

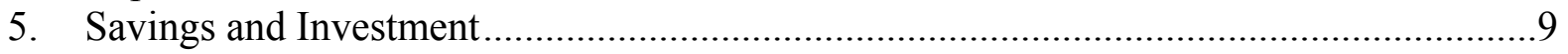

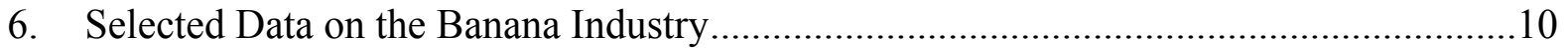

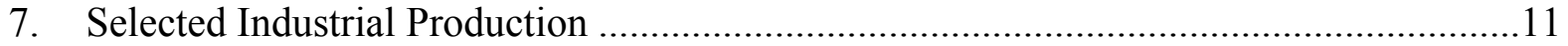

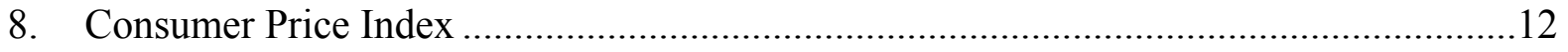

9. Operations of the Consolidated Public Sector (In percent of GDP) ……….....................13

10. Operations of the Central Government (In percent of GDP) ...........................................14

11. Operations of the Central Government (In millions of EC\$) ..........................................15

12. Consolidated Accounts of the Nonfinancial Public Enterprises ........................................16

13. Expenditure and Financing of the Public Sector Investment Program (PSIP) (In percent of GDP) …………………….................................17

14. Expenditure and Financing of the Public Sector Investment

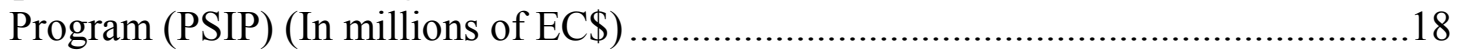

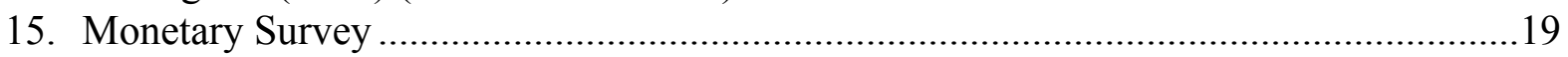

16. Summary Operations of the Eastern Caribbean Central Bank ………………..................2

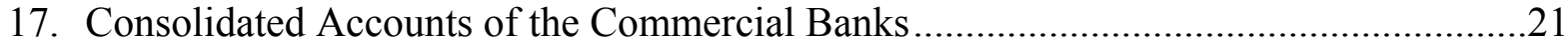

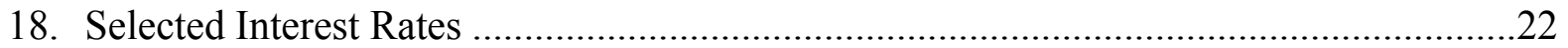

19. Sectoral Distribution of Commercial Bank Loans and Advances.....................................23

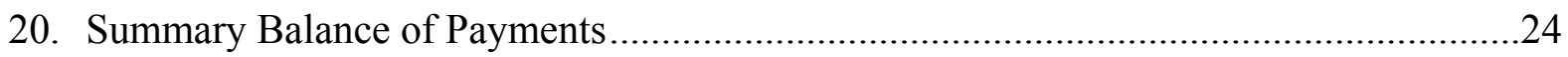

21. Value, Volume and Unit Value of Major Exports......................................................2.

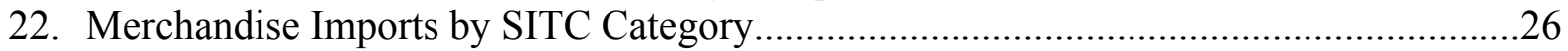




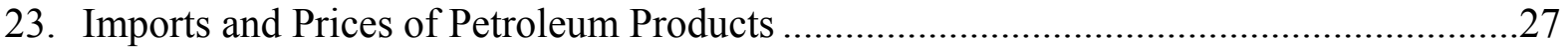

24. Merchandise Trade Volumes, Unit Values and Terms of Trade.................................28

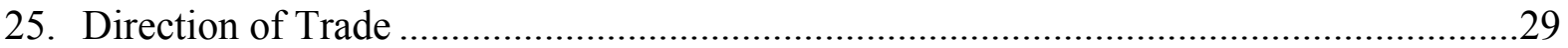

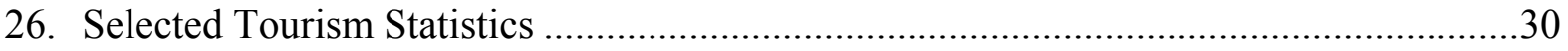

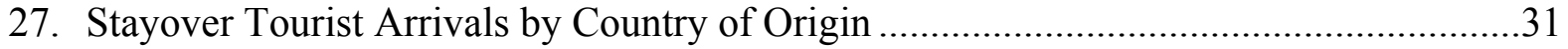

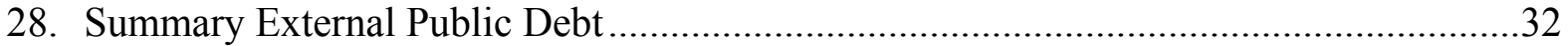

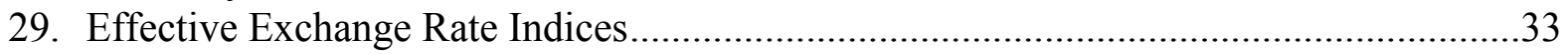

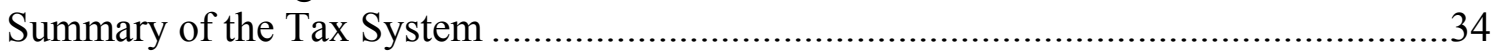




\section{St. Lucia: Basic Data}

\section{Social and Demographic Indicators}

Area (sq. km.)

Arable land (percent of land area, 2000)

Population (2001)

Total (thousands)

Annual rate of growth, 1991-2001

(percent a year)

GDP per capita (US\$), 2001

Population characteristics (2001)

Infant mortality (per thousand live births)

Distribution of labor force (in percent, 1999)

Agriculture

Industry and mining

Services

$\begin{array}{rlr}616 & \text { Health(1998) } & \\ 4.9 & \text { Population per physician } & 2,533 \\ & \text { Population per hospital bed } & 292 \\ & & \\ 157.9 & \text { Access to safe water (1998) } \\ & \text { Percent of population } \\ 1.4 & \text { Urban } & 100.0 \\ 257 & \text { Rural } & \ldots \\ 4,142 & & \\ & \text { Education }(2001) & 24,719 \\ 16.8 & \text { Gross enrollment } & 13,265 \\ & \text { Primary } & \\ 21.7 & \text { Secondary } & \\ 19.2 & \text { GDP }(2001) & \text { EC } \$ 1,766 \text { million } \\ 59.0 & & \text { US } \$ 654 \text { million }\end{array}$

II. Economic Indicators, 1999-2003

0.0

19
Density (per sq. km.)

(In percent of GDP)
Origin of GDP

Agriculture and mining

Manufacturing and construction

Services

$\begin{array}{rr}7.9 & 7 \\ 14.6 & 13 \\ 77.5 & 78 .\end{array}$

(Annual percentage changes, unless otherwise indicated)
National accounts and prices
Real GDP

Real GDP per capita

Consumer price index (period average)

Consumer price index (end of period)

Unemployment rate (in percent)
GDP deflator

$\begin{array}{rrrrr}2.8 & 0.1 & -4.3 & 0.0 & 2.3 \\ 1.6 & -1.3 & -5.5 & -1.4 & 0.9 \\ 4.7 & 2.2 & 1.3 & 1.2 & 0.6 \\ 3.5 & 3.6 & 2.1 & -0.2 & 1.0 \\ 6.1 & 0.4 & 2.1 & -0.6 & 0.5 \\ 18.1 & 16.4 & 18.1 & 16.2 & \ldots\end{array}$

(Ratios to GDP)

Public finances 1/

Central government

Total revenue 2 /

Total expenditure

Of which

Interest

Savings

Primary balance 3 /

Overall balance 3/

2000

2001

2002

Consolidated public sector

Savings

Primary balance 3 /

Overall balance 3/

Total public debt

$\begin{array}{rrrrr}29.2 & 27.0 & 25.7 & 26.0 & 26.6 \\ 28.7 & 28.3 & 30.1 & 33.5 & 32.4 \\ & & & & \\ 1.3 & 1.6 & 2.4 & 2.5 & 3.3 \\ 6.1 & 5.5 & 1.6 & 0.3 & 0.0 \\ 1.8 & 0.3 & -2.0 & -5.0 & -2.5 \\ 0.5 & -1.3 & -4.4 & -7.5 & -5.8 \\ & & & & \\ 10.7 & 10.2 & 5.6 & 4.3 & 4.3 \\ 4.5 & 2.9 & 1.3 & -1.4 & 1.0 \\ 2.6 & 0.6 & -1.7 & -4.4 & -2.8 \\ 38.7 & 43.9 & 49.4 & 61.6 & 65.7\end{array}$

7.9

78.6
7.2

13.2

79.6

5.8

12.2

82.0

\section{3}

0.6

0.5

..

(12-month percentage changes, unless otherwise indicated)

Money and credit 4/

Liabilities to private sector

Of which

Money

Quasi money

Net domestic assets of the banking system

Of which

Credit to the public sector (net)

Credit to the private sector

Liabilities to private sector, in percent of GDP
9.7

8.0

10.3

10.8

$-2.9$

13.6

60.5 
St. Lucia: Basic Data

\begin{tabular}{|c|c|c|c|c|c|}
\hline & 1999 & 2000 & 2001 & 2002 & $\begin{array}{r}\text { Est. } \\
2003 \\
\end{array}$ \\
\hline \multicolumn{6}{|c|}{ (In millions of U.S. dollars, unless otherwise indicated) } \\
\hline \multicolumn{6}{|l|}{ Balance of payments } \\
\hline Current account & -83.6 & -84.8 & -59.0 & -84.9 & -129.1 \\
\hline Trade balance (goods) & -251.1 & -259.6 & -218.0 & -205.7 & -286.4 \\
\hline Exports, f.o.b. & 60.9 & 52.9 & 54.1 & 69.8 & 66.1 \\
\hline Imports, f.o.b. & -312.0 & -312.5 & -272.1 & -275.6 & -352.6 \\
\hline Services and transfers (net) & 167.5 & 174.8 & 158.9 & 120.9 & 157.3 \\
\hline \multicolumn{6}{|l|}{ Of which } \\
\hline Tourism receipts & 277.1 & 286.1 & 251.2 & 230.7 & 266.7 \\
\hline Interest (public sector) & 6.9 & 8.1 & 11.5 & 10.7 & 15.5 \\
\hline Capital and financial account 5/ & 91.3 & 93.0 & 69.2 & 90.0 & 146.3 \\
\hline Capital transfers & 25.1 & 15.8 & 26.4 & 21.4 & 25.9 \\
\hline Official borrowing (net) & 13.3 & 29.5 & 20.9 & 41.5 & 67.5 \\
\hline Private capital 5/ & 52.9 & 47.8 & 21.8 & 27.1 & 52.9 \\
\hline Change in net international reserves (increase -) 6/ & -7.7 & -8.2 & -10.1 & -5.1 & -17.2 \\
\hline Exports (in percent of GDP) & 9.1 & 7.7 & 8.3 & 10.3 & 9.5 \\
\hline Imports (in percent of GDP) & 46.6 & 45.7 & 41.6 & 40.7 & 50.9 \\
\hline Current account (in percent of GDP) & -12.5 & -12.4 & -9.0 & -12.5 & -18.6 \\
\hline Goods exports (in US\$, annual percentage change) & -13.5 & -13.2 & 2.4 & 29.1 & -5.3 \\
\hline Goods imports (in US\$, annual percentage change) & 5.0 & -1.8 & -13.0 & 4.4 & 20.0 \\
\hline Terms of trade (annual percentage change) $7 /$ & 7.1 & -5.9 & 1.6 & 1.2 & 3.0 \\
\hline Real effective exchange rate (12-month percentage change) $8 /$ & 8.5 & 3.0 & 0.3 & -6.2 & -11.5 \\
\hline \multicolumn{6}{|l|}{ International reserve position and external debt } \\
\hline Gross official reserves & 79.2 & 83.1 & 91.2 & 95.9 & 112.1 \\
\hline Net official reserves (Central Bank, imputed) & 72.5 & 77.0 & 87.1 & 91.9 & 109.1 \\
\hline Net reserves of the banking system & -42.3 & -33.8 & -43.6 & -52.9 & -17.8 \\
\hline \multicolumn{6}{|l|}{ Outstanding external debt, in percent of GDP } \\
\hline Public 9/ & 25.4 & 28.8 & 32.8 & 38.4 & 47.6 \\
\hline Private & $\ldots$ & $\ldots$ & $\ldots$ & $\ldots$ & $\ldots$ \\
\hline \multicolumn{6}{|l|}{ Total debt service ratio (in percent of exports of goods } \\
\hline and services) & 4.4 & 5.3 & 10.4 & 10.0 & 8.5 \\
\hline Gross reserves/short-term debt (in percent) & $\ldots$ & $\ldots$ & $\ldots$ & $\ldots$ & $\ldots$ \\
\hline \multicolumn{6}{|l|}{ IMF data (as of December 31, 2003) } \\
\hline Membership status: & & & & & cle VIII \\
\hline Intervention currency and rate & & & U.S. dollar at & EC $\$ 2.70$ & dollar \\
\hline Quota & & & & & million \\
\hline Fund holdings of Eastern Caribbean dollars & & & & SD & million \\
\hline Outstanding purchases and loans & & & & & None \\
\hline \multicolumn{6}{|l|}{ SDR department } \\
\hline Net cumulative allocation & & & & SD & million \\
\hline Holdings & & & & & million \\
\hline
\end{tabular}

Sources: St. Lucian authorities; World Development Indicators; and Fund staff estimates.

1/ Data for fiscal years beginning April. The Public Sector comprises the Central Government, the Castries City Council, the National Insurance Corporation, the St. Lucia Air and Sea Ports Authority, the Water and Sewerage Company, the National Development Corporation, and the Marketing Board.

2/ Including grants.

3/ After grants.

4/ Change as a percentage of liabilities to the private sector at the beginning of the period.

$5 /$ Includes errors and omissions.

6/ Imputed reserves at the ECCB.

7/ Excluding tourism.

8/ November for 2003.

9/ Total public and publicly guaranteed debt. 
Table 1. St. Lucia: Selected Economic and Financial Indicators

\begin{tabular}{|c|c|c|c|c|c|}
\hline & 1999 & 2000 & 2001 & 2002 & $\begin{array}{r}\text { Est. } \\
2003 \\
\end{array}$ \\
\hline \multicolumn{6}{|c|}{ (Annual percentage changes, unless otherwise specified) } \\
\hline \multicolumn{6}{|l|}{ Output and prices } \\
\hline Real GDP at factor cost & 2.8 & 0.1 & -4.3 & 0.0 & 2.3 \\
\hline GDP at current market prices & 6.1 & 2.1 & -4.3 & 3.4 & 2.4 \\
\hline GDP deflator at factor cost & 4.7 & 2.2 & 1.3 & 1.2 & 0.6 \\
\hline Consumer prices (end of period) & 6.1 & 0.4 & 2.1 & -0.6 & 0.5 \\
\hline Unemployment rate & 18.1 & 16.4 & 18.1 & 16.2 & \\
\hline Banana production & -10.7 & 7.7 & -51.6 & 41.5 & -34.8 \\
\hline Tourist stayovers & 3.3 & 3.6 & -7.3 & 1.3 & 11.0 \\
\hline \multicolumn{6}{|l|}{ External sector } \\
\hline Exports, f.o.b. & -13.5 & -13.2 & 2.4 & 29.1 & -5.3 \\
\hline Imports, f.o.b. & 5.8 & 0.1 & -12.9 & 1.3 & 27.9 \\
\hline Travel receipts & 1.9 & 3.3 & -12.2 & -8.1 & 15.6 \\
\hline Terms of trade & 7.3 & -7.0 & -3.9 & -6.5 & 1.7 \\
\hline Excluding tourism & 7.1 & -5.9 & 1.6 & 1.2 & 3.0 \\
\hline $\begin{array}{l}\text { Nominal effective exchange rate (end of } \\
\text { period, depreciation -) } 1 /\end{array}$ & 4.6 & 6.4 & 1.8 & -5.3 & -4.7 \\
\hline Real effective exchange rate (end of & 85 & 30 & 03 & 62 & 76 \\
\hline \multicolumn{6}{|l|}{ Money and credit 2/ } \\
\hline Domestic assets (net) & 10.8 & 4.7 & 4.6 & 4.1 & -6.3 \\
\hline Credit to public sector (net) & -2.9 & -1.8 & -2.0 & 2.5 & -2.5 \\
\hline Credit to private sector & 13.6 & 8.0 & 5.1 & 0.9 & -4.1 \\
\hline Money and quasi-money & 9.7 & 7.9 & 4.7 & 3.2 & 4.8 \\
\hline Velocity of money (M2) 3/ & 1.7 & 1.6 & 1.5 & 1.5 & 1.4 \\
\hline \multicolumn{6}{|c|}{ (In percent of GDP, unless otherwise specified) } \\
\hline \multicolumn{6}{|l|}{ Nonfinancial public sector 4 / } \\
\hline Current balance & 7.7 & 7.0 & 2.8 & 1.1 & 1.1 \\
\hline Of which: central government & 6.1 & 5.5 & 1.6 & 0.3 & 0.0 \\
\hline Capital outlays & 11.4 & 9.0 & 8.4 & 10.4 & 8.9 \\
\hline Overall balance (before grants) & -3.5 & -1.9 & -5.4 & -9.4 & -7.9 \\
\hline Overall balance (after grants) & -0.1 & -1.3 & -4.0 & -7.4 & -5.7 \\
\hline Of which: central government & 0.5 & -1.3 & -4.4 & -7.5 & -5.8 \\
\hline Overall balance (after grants), including & & & & & \\
\hline the National Insurance Corporation (NIC) & 2.6 & 0.6 & -1.7 & -4.4 & -2.8 \\
\hline Total public sector debt $5 /$ & 38.7 & 43.9 & 49.4 & 61.6 & 65.7 \\
\hline Of which: central government & 26.4 & 31.0 & 36.0 & 48.4 & 52.7 \\
\hline \multicolumn{6}{|l|}{ External sector } \\
\hline Current account balance & -12.5 & -12.4 & -9.0 & -12.5 & -18.6 \\
\hline External debt (end of period) 6/ & 25.4 & 28.8 & 32.8 & 38.4 & 47.6 \\
\hline Debt-service ratio $7 /$ & 4.4 & 5.3 & 10.4 & 10.0 & 8.5 \\
\hline \multicolumn{6}{|c|}{ (In millions of U.S. dollars) } \\
\hline GDP at current market prices & 669.0 & 683.2 & 654.0 & 676.5 & 692.8 \\
\hline $\begin{array}{l}\text { Change in net international } \\
\text { reserves (increase -) 8/ }\end{array}$ & -3.9 & -4.4 & -17.0 & -13.7 & -4.0 \\
\hline
\end{tabular}

Sources: St. Lucian authorities; ECCB; and Fund staff estimates and projections.

1/ For 2003, 12-month change through November 2003.

2/ Changes in relation to liabilities to private sector at beginning of period.

3 / Nominal GDP at market prices divided by the average stock of money (measured as the simple average of the current period stock and the stock 12-months earlier).

4/ Data are for fiscal years beginning April 1. The nonfinancial public sector is defined here as the central government, the Castries City Council, the St. Lucia Air and Sea Ports Authority, the Water and Sewerage Company, the National Development Corporation, and the Marketing Board.

5/ Includes liabilities to the NIC.

6/ Total public and publicly guaranteed debt.

$7 /$ In percent of exports of goods and services.

8/ Imputed reserves at the ECCB. 
Table 2. St. Lucia: GDP By Economic Activity at Constant Factor Cost

\begin{tabular}{|c|c|c|c|c|c|}
\hline & 1999 & 2000 & 2001 & 2002 & $\begin{array}{r}\text { Est. } \\
2003 \\
\end{array}$ \\
\hline \multicolumn{6}{|c|}{ ( In millions of 1990 Eastern Caribbean dollars ) } \\
\hline Gross domestic product & $1,167.9$ & $1,169.5$ & $1,118.9$ & $1,118.9$ & $1,144.9$ \\
\hline Primary sector & 90.6 & 93.2 & 70.6 & 65.2 & 58.2 \\
\hline Agriculture & 83.9 & 85.6 & 64.9 & 59.4 & 52.4 \\
\hline Bananas & 41.1 & 43.7 & 22.4 & 26.5 & 20.1 \\
\hline Other crops & 21.1 & 19.6 & 20.5 & 13.5 & 12.8 \\
\hline Livestock & 6.7 & 7.7 & 7.2 & 6.9 & 6.6 \\
\hline Fishing & 13.5 & 13.2 & 13.6 & 11.5 & 11.9 \\
\hline Forestry & 1.6 & 1.4 & 1.2 & 1.1 & 0.9 \\
\hline Mining and quarrying & 6.7 & 7.7 & 5.7 & 5.8 & 5.8 \\
\hline Secondary sector & 239.8 & 236.4 & 232.2 & 228.6 & 226.0 \\
\hline Manufacturing & 71.8 & 69.9 & 66.5 & 69.8 & 70.2 \\
\hline Construction & 109.2 & 104.2 & 99.0 & 94.2 & 89.3 \\
\hline Electricity, gas, and water & 58.8 & 62.4 & 66.7 & 64.6 & 66.5 \\
\hline Services & 936.9 & 944.0 & 922.3 & 933.2 & 970.3 \\
\hline Wholesale and retail trade & 156.2 & 146.1 & 125.2 & 126.8 & 133.1 \\
\hline Hotels and restaurants & 151.6 & 155.6 & 139.2 & 138.4 & 151.7 \\
\hline Transport and communications & 230.9 & 234.9 & 246.0 & 250.3 & 260.4 \\
\hline Banking and insurance & 120.8 & 124.4 & 127.7 & 129.3 & 131.3 \\
\hline Real estate and housing & 81.5 & 83.4 & 87.2 & 89.3 & 91.5 \\
\hline Government services & 140.3 & 142.1 & 143.7 & 145.0 & 148.0 \\
\hline Other services & 55.7 & 57.6 & 53.3 & 54.3 & 54.3 \\
\hline Less imputed banking charges & 99.5 & 104.1 & 106.2 & 108.2 & 109.6 \\
\hline \multicolumn{6}{|c|}{ (Annual percentage change) } \\
\hline Gross domestic product & 2.8 & 0.1 & -4.3 & 0.0 & 2.3 \\
\hline Primary sector & -15.8 & 2.9 & -24.2 & -7.6 & -10.8 \\
\hline Agriculture & -17.6 & 2.0 & -24.1 & -8.5 & -11.9 \\
\hline Bananas & -25.4 & 6.4 & -48.8 & 18.6 & -24.2 \\
\hline Other crops & -17.3 & -7.4 & 4.6 & -34.1 & -5.0 \\
\hline Livestock & -26.9 & 15.0 & -6.1 & -4.7 & -3.2 \\
\hline Fishing & 29.3 & -1.8 & 3.3 & -16.0 & 3.7 \\
\hline Forestry & -10.2 & -11.0 & -12.4 & -14.2 & -16.4 \\
\hline Mining and quarrying & 15.3 & 14.0 & -25.5 & 1.9 & 0.3 \\
\hline Secondary sector & 6.9 & -1.4 & -1.8 & -1.5 & -1.1 \\
\hline Manufacturing & 4.2 & -2.6 & -4.9 & 5.0 & 0.6 \\
\hline Construction & 12.8 & -4.6 & -5.0 & -4.8 & -5.2 \\
\hline Electricity, gas, and water & 0.2 & 6.1 & 7.0 & -3.2 & 3.0 \\
\hline Services & 4.4 & 0.8 & -2.3 & 1.2 & 4.0 \\
\hline Wholesale and retail trade & 3.0 & -6.5 & -14.3 & 1.2 & 5.0 \\
\hline Hotels and restaurants & 4.2 & 2.7 & -10.5 & -0.6 & 9.7 \\
\hline Transport and communications & 5.6 & 1.7 & 4.7 & 1.7 & 4.1 \\
\hline Banking and insurance & 8.8 & 3.0 & 2.7 & 1.2 & 1.5 \\
\hline Real estate and housing & 3.6 & 2.4 & 4.5 & 2.5 & 2.5 \\
\hline Government services & 1.2 & 1.3 & 1.1 & 0.9 & 2.1 \\
\hline Other services & 3.2 & 3.3 & -7.3 & 1.8 & 0.0 \\
\hline Less imputed banking charges & 6.1 & 4.7 & 2.0 & 1.9 & 1.3 \\
\hline
\end{tabular}

Sources: Statistics Department of the Ministry of Finance; and Fund staff estimates. 
Table 3. St. Lucia: GDP By Economic Activity at Current Factor Cost

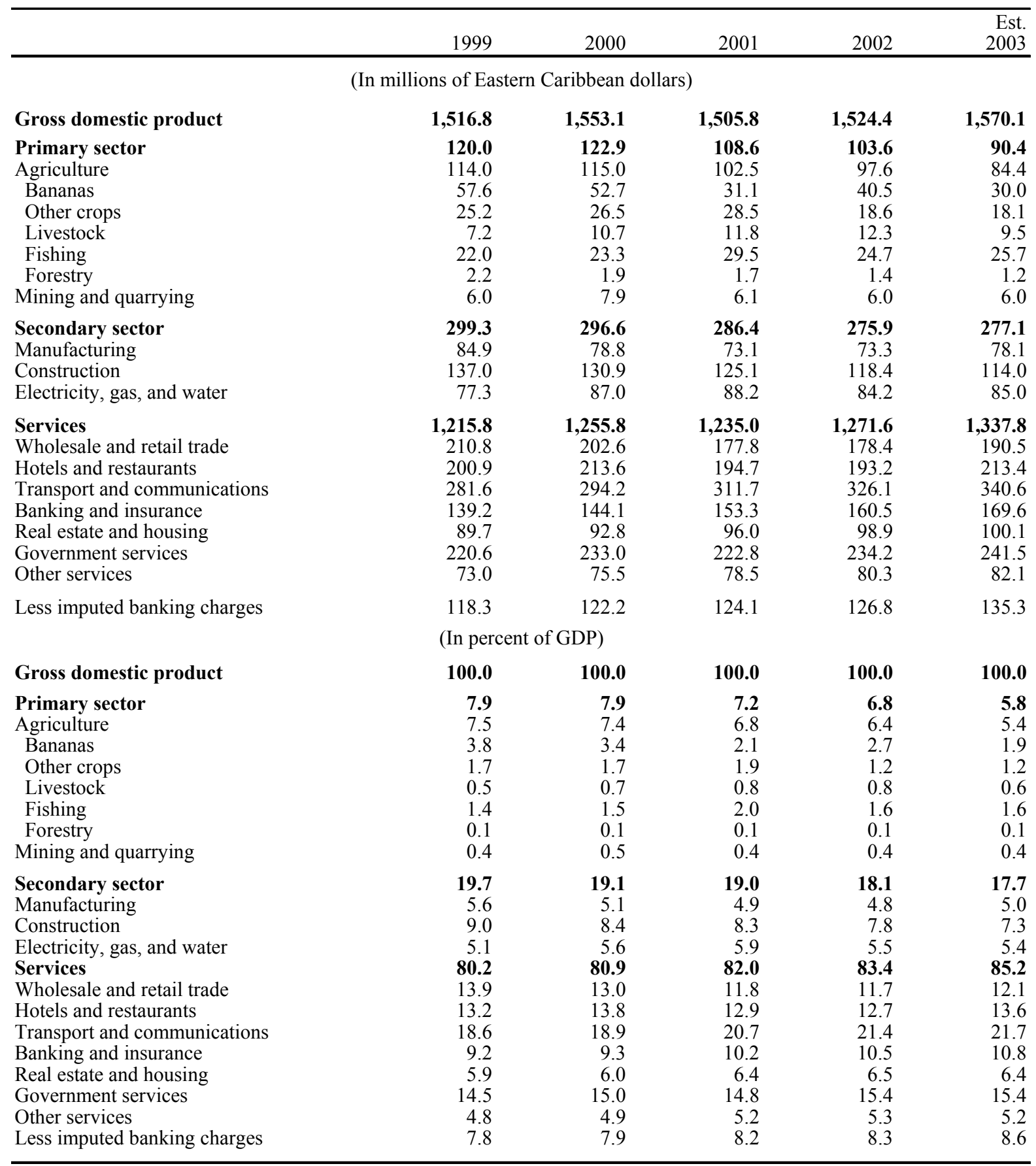

Sources: Statistics Department of the Ministry of Finance; and Fund staff estimates. 
Table 4: St. Lucia: Expenditure on GDP at Current Prices

\begin{tabular}{|c|c|c|c|c|c|}
\hline & 1999 & 2000 & 2001 & 2002 & $\begin{array}{r}\text { Est. } \\
2003 \\
\end{array}$ \\
\hline \multicolumn{6}{|c|}{ (In millions of Eastern Caribbean dollars) } \\
\hline Consumption expenditure & $1,476.5$ & $1,523.9$ & $1,475.8$ & $1,519.5$ & $1,585.3$ \\
\hline Public sector $1 /$ & 408.7 & 430.1 & 489.5 & 492.1 & 516.3 \\
\hline Private sector & $1,067.8$ & $1,093.8$ & 986.3 & $1,027.4$ & $1,069.0$ \\
\hline Gross domestic investment & 500.5 & 490.7 & 459.4 & 415.9 & 539.1 \\
\hline Public sector $1 /$ & 191.2 & 175.1 & 152.3 & 181.4 & 174.0 \\
\hline \multicolumn{6}{|l|}{ Of which } \\
\hline Central government & 158.6 & 142.2 & 134.5 & 168.4 & 157.7 \\
\hline Private sector & 309.3 & 315.6 & 306.1 & 234.5 & 365.1 \\
\hline Gross domestic expenditure & $1,976.9$ & $2,014.6$ & $1,935.2$ & $1,935.4$ & $2,124.4$ \\
\hline Balance of goods and nonfactor services & -170.5 & -170.0 & -169.5 & -108.8 & -253.9 \\
\hline Exports of goods and nonfactor services & $1,031.0$ & 981.0 & 961.0 & 845.0 & $1,042.2$ \\
\hline Imports of goods and nonfactor services & $1,202.0$ & $1,151.0$ & $1,131.0$ & 954.0 & $1,296.2$ \\
\hline GDP at market prices & $1,806.4$ & $1,844.7$ & $1,765.7$ & $1,826.6$ & $1,870.5$ \\
\hline Net factor income & -103.8 & -116.4 & -114.9 & -117.2 & -132.4 \\
\hline GNP at market prices & $1,702.6$ & $1,728.3$ & $1,650.9$ & $1,709.3$ & $1,738.1$ \\
\hline \multicolumn{6}{|c|}{ (In percent of GDP) } \\
\hline Consumption expenditure & 81.7 & 82.6 & 83.6 & 83.2 & 84.8 \\
\hline Public sector & 22.6 & 23.3 & 27.7 & 26.9 & 27.6 \\
\hline Private sector & 59.1 & 59.3 & 55.9 & 56.2 & 57.2 \\
\hline Gross domestic investment & 27.7 & 26.6 & 26.0 & 22.8 & 28.8 \\
\hline Public sector & 10.6 & 9.5 & 8.7 & 9.9 & 9.3 \\
\hline \multicolumn{6}{|l|}{ Of which } \\
\hline Central government & 8.8 & 7.7 & 7.6 & 9.2 & 8.4 \\
\hline Private sector & 17.1 & 17.1 & 17.3 & 12.8 & 19.5 \\
\hline Gross domestic expenditure & 109.4 & 109.2 & 109.6 & 106.0 & 113.6 \\
\hline Balance of goods and nonfactor services & -9.4 & -9.2 & -9.6 & -6.0 & -13.6 \\
\hline Exports of goods and nonfactor services & 57.1 & 53.2 & 54.4 & 46.2 & 55.7 \\
\hline Imports of goods and nonfactor services & 66.5 & 62.4 & 64.0 & 55.2 & 69.3 \\
\hline GDP at market prices & 100.0 & 100.0 & 100.0 & 100.0 & 100.0 \\
\hline Net indirect taxes & 16.0 & 15.8 & 14.7 & 16.5 & 16.1 \\
\hline GDP at factor cost & 84.0 & 84.2 & 85.3 & 83.5 & 83.9 \\
\hline \multicolumn{6}{|l|}{ Memorandum item: } \\
\hline GDP at market price (annual percentage change) & 6.1 & 2.1 & -4.3 & 3.5 & 2.4 \\
\hline
\end{tabular}

Sources: Statistics Department of the Ministry of Finance; and Fund staff estimates.

1/ The public sector comprises the central government, the Castries City Council, the National Insurance Corporation, the St. Lucia Air and Sea Ports Authority, the Water and Sewerage Company, the National Development Corporation, and the Marketing Board. 
Table 5: St. Lucia: Savings and Investment

\begin{tabular}{|c|c|c|c|c|c|}
\hline & 1999 & 2000 & 2001 & 2002 & $\begin{array}{r}\text { Est. } \\
2003 \\
\end{array}$ \\
\hline \multicolumn{6}{|c|}{ (In millions of Eastern Caribbean dollars) } \\
\hline Gross national saving & 274.8 & 261.7 & 300.0 & 186.7 & 190.5 \\
\hline Public 1/ & 133.6 & 130.1 & 68.5 & 26.8 & 19.7 \\
\hline Private & 141.2 & 131.6 & 231.5 & 159.8 & 170.8 \\
\hline External saving & 225.6 & 229.0 & 159.4 & 229.1 & 348.7 \\
\hline Total saving & 500.5 & 490.7 & 459.4 & 415.8 & 539.1 \\
\hline Gross domestic investment & 500.5 & 490.7 & 459.4 & 415.8 & 539.1 \\
\hline Public sector $1 /$ & 191.2 & 175.1 & 153.3 & 181.4 & 174.0 \\
\hline Of which & & & & & \\
\hline Central government & 158.6 & 142.2 & 134.5 & 168.4 & 157.7 \\
\hline Private Sector & 309.3 & 315.6 & 306.1 & 234.4 & 365.1 \\
\hline \multicolumn{6}{|c|}{ (In percent of GDP) } \\
\hline Gross national saving & 15.2 & 14.2 & 17.0 & 10.2 & 10.2 \\
\hline Public & 7.4 & 7.1 & 3.9 & 1.5 & 1.1 \\
\hline Private & 7.8 & 7.1 & 13.1 & 8.8 & 9.1 \\
\hline External saving & 12.5 & 12.4 & 9.0 & 12.5 & 18.6 \\
\hline Total saving & 27.7 & 26.6 & 26.0 & 22.8 & 28.8 \\
\hline Gross domestic investment & 27.7 & 26.6 & 26.0 & 22.8 & 28.8 \\
\hline Public sector & 10.6 & 9.5 & 8.7 & 9.9 & 9.3 \\
\hline \multicolumn{6}{|l|}{ Of which } \\
\hline Central government & 8.8 & 7.7 & 7.6 & 9.2 & 8.4 \\
\hline Private sector & 17.1 & 17.1 & 17.3 & 12.8 & 19.5 \\
\hline
\end{tabular}

Sources: Statistics Department of the Ministry of Finance; and Fund staff estimates.

1/ The public sector comprises the central government, the Castries City Council, the National Insurance Corporation, the St. Lucia Air and Sea Ports Authority, the Water and Sewerage Company, the National Development Corporation, and the Marketing Board. 
Table 6. St. Lucia: Selected Data on the Banana Industry

\begin{tabular}{|c|c|c|c|c|c|}
\hline & 1999 & 2000 & 2001 & 2002 & $\begin{array}{r}\text { Est. } \\
2003 \\
\end{array}$ \\
\hline Banana exports (000 tons) & 65.2 & 70.3 & 34.0 & 48.2 & 31.4 \\
\hline $\begin{array}{l}\text { Banana export receipts } \\
\text { (in millions of EC\$) }\end{array}$ & 87.0 & 86.0 & 41.2 & 58.6 & 41.6 \\
\hline $\begin{array}{l}\text { Export unit price } \\
\text { (in US\$/000 tons) }\end{array}$ & 493.8 & 453.0 & 447.8 & 450.7 & 487.3 \\
\hline \multicolumn{6}{|c|}{ (In percent) } \\
\hline Memorandum items: & & & & & \\
\hline Banana exports/GDP & 4.8 & 4.7 & 2.3 & 3.2 & 2.2 \\
\hline Banana exports/goods exports & 52.9 & 60.2 & 28.2 & 31.1 & 23.1 \\
\hline Banana exports/exports of goods and NFS & 8.4 & 8.3 & 4.3 & 6.3 & 4.0 \\
\hline
\end{tabular}

Sources: Windward Islands Banana Development and Export Company (WIBDECO); and Fund staff estimates. 
Table 7. St. Lucia: Selected Industrial Production

(Values in thousands of Eastern Caribbean dollars; quantity as indicated)

\begin{tabular}{|c|c|c|c|c|c|}
\hline & 1999 & 2000 & 2001 & 2002 & $\begin{array}{r}\text { Est } \\
2003 \\
\end{array}$ \\
\hline \multicolumn{6}{|l|}{ Copra } \\
\hline Value & 2,924 & 1,899 & 2,124 & 1,270 & 1,308 \\
\hline Tons & 2,900 & 1,884 & 2,189 & 1,138 & 1,162 \\
\hline \multicolumn{6}{|c|}{ Unrefined coconut oil } \\
\hline Value & 3,283 & 2,552 & 3,624 & 70 & 0 \\
\hline Liters $(000$ 's) & 1,379 & 1,072 & 1304 & 734 & 0 \\
\hline \multicolumn{6}{|c|}{ Refined coconut oil } \\
\hline Value & 3,526 & 3,378 & 2,591 & 151 & 177 \\
\hline Liters $(000 ' \mathrm{~s})$ & 888 & 851 & 725 & 2,040 & 2,288 \\
\hline \multicolumn{6}{|l|}{ Coconut meal } \\
\hline Value & 316 & 246 & 234 & 145 & 145 \\
\hline Kgs. $(000 ' s)$ & 717 & 1,226 & 1,172 & 727 & 829 \\
\hline \multicolumn{6}{|c|}{ Food, beverages, and tobacco } \\
\hline Value & 53,661 & 49,254 & 51,769 & 59,606 & 64,158 \\
\hline \multicolumn{6}{|c|}{ Paper products and cardboard boxes } \\
\hline Value & 23,265 & 22,098 & 16,548 & 21,210 & 15,125 \\
\hline Quantity (000's) & 23,815 & 19,034 & 7,977 & 14,164 & 7,928 \\
\hline \multicolumn{6}{|c|}{ Electrical products } \\
\hline Value & 23,753 & 31,412 & 18,342 & 26,840 & 20,846 \\
\hline Quantity (000's) & 23,174 & 27,802 & 19,110 & 20,824 & 15,551 \\
\hline \multicolumn{6}{|l|}{ Textile goods } \\
\hline Value & 3,450 & 2,475 & 1,801 & 4,762 & 7,900 \\
\hline Quantity (000's) & 174,888 & 177,413 & 152,247 & 111,871 & 81,815 \\
\hline \multicolumn{6}{|l|}{ Wearing apparel } \\
\hline Value & 7,979 & 3,582 & 1,862 & 1,465 & 956 \\
\hline Doz. Pcs. (000's) & 71 & 24 & 21 & 17 & 11 \\
\hline
\end{tabular}

Source: Statistics Department of the Ministry of Finance. 
Table 8. St. Lucia: Consumer Price Index (April 1984=100)

\begin{tabular}{lccccc}
\hline & 1999 & 2000 & 2001 & 2002 & 2003 \\
\hline & (Period averages) & & & & \\
All items & $\mathbf{1 6 1 . 2}$ & $\mathbf{1 6 6 . 9}$ & $\mathbf{1 7 0 . 4}$ & $\mathbf{1 7 0 . 0}$ & $\mathbf{1 7 1 . 8}$ \\
Food & 168.0 & 170.4 & 175.5 & 173.4 & 177.0 \\
Beverages and tobacco & 143.1 & 147.0 & 153.0 & 157.0 & 159.2 \\
Clothing and footwear & 219.6 & 236.4 & 236.9 & 237.0 & 237.0 \\
Housing & 112.6 & 110.4 & 110.8 & 110.8 & 110.7 \\
Fuel and light & 124.1 & 133.0 & 133.8 & 131.9 & 135.6 \\
Furniture, household equipment & 168.9 & 178.2 & 181.4 & 179.2 & 178.0 \\
Health care & 215.0 & 222.1 & 225.3 & 238.8 & 236.1 \\
Transport and communication & 164.4 & 172.4 & 177.7 & 184.0 & 184.4 \\
Recreation and education & 196.0 & 246.3 & 247.0 & 246.6 & 245.8 \\
Miscellaneous goods and services & 145.8 & 152.3 & 152.9 & 154.2 & 153.9
\end{tabular}

(Annual percentage change)

All items
Food
Beverages and tobacco
Clothing and footwear
Housing
Fuel and light
Furniture, household equipment
Health care
Transport and communication
Recreation and education
Miscellaneous goods and services

\section{All items}

Food

Beverages and tobacco

Clothing and footwear

Housing

Fuel and light

Furniture, household equipment

Health care

Transport and communication

Recreation and education

Miscellaneous goods and services

$\begin{array}{rr}\mathbf{3 . 5} & \mathbf{3 . 6} \\ 2.9 & 1.4 \\ 0.9 & 2.7 \\ 5.5 & 7.7 \\ 0.0 & -2.0 \\ 0.6 & 7.2 \\ 6.0 & 5.5 \\ 8.3 & 3.3 \\ 0.2 & 4.9 \\ 13.4 & 25.7 \\ 5.5 & 4.5\end{array}$

(End of period)

\section{(Percentage change during year)}

\begin{tabular}{lrrrrr} 
All items & $\mathbf{6 . 1}$ & $\mathbf{0 . 4}$ & $\mathbf{2 . 1}$ & $\mathbf{- 0 . 6}$ & $\mathbf{0 . 5}$ \\
Food & 5.2 & -2.7 & 3.6 & -1.8 & 1.8 \\
Beverages and tobacco & 2.7 & 1.8 & 5.2 & 2.3 & 0.2 \\
Clothing and footwear & 14.0 & 0.1 & 0.3 & 0.0 & 0.0 \\
Housing & 0.0 & -1.6 & 0.0 & -0.1 & 0.0 \\
Fuel and light & 5.8 & 9.3 & -7.4 & 3.1 & 0.7 \\
Furniture, household equipment & 9.1 & 2.0 & 0.0 & -1.9 & 0.0 \\
Health care & 6.1 & 2.4 & 10.8 & -1.0 & -3.9 \\
Transport and communication & 0.5 & 7.4 & 1.0 & 5.4 & -5.3 \\
Recreation and education & 19.4 & 17.6 & 0.0 & -0.7 & 0.2 \\
Miscellaneous goods and services & 7.3 & 1.2 & 1.8 & -1.6 & 1.0 \\
& & & & & \\
\hline
\end{tabular}

Source: Statistics Department of Ministry of Finance. 
Table 9. St. Lucia: Operations of the Consolidated Public Sector 1/ 2/

(In percent of GDP)

\begin{tabular}{|c|c|c|c|c|c|}
\hline & $1998 / 99$ & $1999 / 00$ & $2000 / 01$ & $2001 / 02$ & $2002 / 03$ \\
\hline Total revenue and grants & 38.7 & 39.3 & 38.0 & 36.3 & 36.7 \\
\hline Current revenue & 35.1 & 35.7 & 37.3 & 34.6 & 34.7 \\
\hline Tax revenue & 23.6 & 23.1 & 23.8 & 21.6 & 22.1 \\
\hline Non-tax revenue & 6.8 & 7.4 & 8.1 & 7.6 & 7.5 \\
\hline Operational surplus of public enterprises & 4.7 & 5.2 & 5.4 & 5.4 & 5.2 \\
\hline Capital revenue & 0.4 & 0.2 & 0.2 & 0.2 & 0.0 \\
\hline Capital grants & 3.3 & 3.4 & 0.6 & 1.5 & 2.0 \\
\hline Total expenditure & 33.7 & 36.7 & 37.4 & 38.0 & 41.2 \\
\hline $\begin{array}{l}\text { Current expenditure } 3 / \\
\text { Of which }\end{array}$ & 25.3 & 25.0 & 27.1 & 29.0 & 30.4 \\
\hline Wages and salaries & 13.2 & 13.4 & 13.8 & 14.3 & 13.9 \\
\hline Interest & 1.8 & 1.9 & 2.3 & 3.0 & 3.0 \\
\hline Capital expenditure & 8.4 & 11.7 & 10.3 & 9.0 & 10.8 \\
\hline Central government & 7.8 & 9.2 & 7.3 & 7.6 & 9.8 \\
\hline Rest of general government & 0.0 & 0.3 & 1.3 & 0.6 & 0.3 \\
\hline Public enterprises & 0.5 & 2.2 & 1.7 & 0.8 & 0.7 \\
\hline Current balance & 9.7 & 10.7 & 10.2 & 5.6 & 4.3 \\
\hline Central government & 6.3 & 6.1 & 5.5 & 1.6 & 0.3 \\
\hline Rest of general government & 3.0 & 2.9 & 3.3 & 2.8 & 3.2 \\
\hline Public enterprises & 0.5 & 1.6 & 1.5 & 1.3 & 0.8 \\
\hline Overall balance before grants & 1.7 & -0.8 & 0.0 & -3.2 & -6.4 \\
\hline $\begin{array}{l}\text { Overall balance after grants } \\
\text { of which: }\end{array}$ & 5.0 & 2.6 & 0.6 & -1.7 & -4.4 \\
\hline Central government & 2.0 & 0.5 & -1.3 & -4.4 & -7.5 \\
\hline National Insurance Corporation & 2.9 & 2.6 & 2.0 & 2.2 & 2.9 \\
\hline Public enterprises & 0.0 & -0.5 & 0.0 & 0.5 & 0.2 \\
\hline
\end{tabular}

Sources: St. Lucian authorities and Fund staff estimates.

1/ Data are for fiscal years beginning April 1.

2/ The public sector comprises the central government, the Castries City Council, The National Insurance Corporation, and four nonfinancial public enterprises.

3/ Refers to Central Government, the Castries City Council and the National Insurance Corporation only, except for interest payments which refer to public enterprises as well. 
Table 10. St. Lucia: Operations of the Central Government 1/ (In percent of GDP)

\begin{tabular}{|c|c|c|c|c|c|}
\hline & 1998/99 & $1999 / 00$ & $2000 / 01$ & $2001 / 02$ & $2002 / 03$ \\
\hline Total revenue and grants & 28.6 & 29.2 & 27.0 & 25.7 & 26.0 \\
\hline Current revenue & 25.0 & 25.6 & 26.4 & 24.1 & 24.0 \\
\hline Tax revenue & 23.1 & 23.1 & 23.8 & 21.6 & 22.1 \\
\hline Taxes on income and profits & 6.1 & 7.0 & 7.6 & 6.6 & 5.7 \\
\hline Taxes on property & 0.1 & 0.0 & 0.1 & 0.2 & 0.2 \\
\hline Taxes on goods and services & 10.9 & 9.4 & 8.3 & 8.6 & 9.5 \\
\hline Consumption taxes & 8.1 & 6.8 & 5.5 & 5.9 & 6.1 \\
\hline Imports & 7.4 & 6.2 & 4.9 & 5.6 & 5.9 \\
\hline Domestic goods & 0.7 & 0.6 & 0.6 & 0.3 & 0.3 \\
\hline Hotel occupancy tax & 1.0 & 1.0 & 1.3 & 1.0 & 1.1 \\
\hline Excises & 0.1 & 0.3 & 0.2 & 0.3 & 0.3 \\
\hline Other & 1.5 & 1.3 & 1.3 & 1.3 & 2.0 \\
\hline Taxes on international trade & 6.1 & 6.6 & 7.8 & 6.1 & 6.6 \\
\hline \multicolumn{6}{|l|}{ Of which } \\
\hline Import duties & 3.9 & 3.9 & 3.5 & 2.9 & 3.1 \\
\hline Service charge (imports) & 1.9 & 1.9 & 1.8 & 1.6 & 1.6 \\
\hline Nontax revenue & 2.0 & 2.6 & 2.7 & 2.5 & 2.0 \\
\hline Capital revenue & 0.3 & 0.1 & $\mathbf{0 . 0}$ & 0.2 & 0.0 \\
\hline Capital grants & 3.2 & 3.4 & 0.6 & 1.5 & 2.0 \\
\hline Total expenditure and net lending & 26.8 & 28.7 & 28.3 & 30.1 & 33.5 \\
\hline Current expenditure & 19.1 & 19.5 & 21.0 & 22.5 & 23.7 \\
\hline Wages and salaries & 10.4 & 10.9 & 11.5 & 11.9 & 11.5 \\
\hline NIS contributions and retirement & 1.3 & 1.5 & 1.9 & 1.9 & 1.9 \\
\hline Goods and services & 3.9 & 3.9 & 4.1 & 4.1 & 4.1 \\
\hline Transfers & 2.3 & 1.9 & 1.9 & 2.2 & 3.7 \\
\hline Interest payments & 1.2 & 1.3 & 1.6 & 2.4 & 2.5 \\
\hline Domestic & 0.8 & 0.9 & 1.2 & 1.3 & 1.2 \\
\hline External & 0.4 & 0.4 & 0.5 & 1.1 & 1.3 \\
\hline Capital expenditure and net lending & 7.7 & 9.2 & 7.3 & 7.6 & 9.8 \\
\hline Current balance & 6.0 & 6.1 & 5.5 & 1.6 & 0.3 \\
\hline Overall balance before grants & -1.5 & -2.9 & -1.9 & -5.9 & -9.5 \\
\hline Overall balance after grants & 1.7 & 0.5 & -1.3 & -4.4 & -7.5 \\
\hline \multicolumn{6}{|l|}{ Memorandum item: } \\
\hline Nominal GDP (in millions of EC dollars) & $1,742.4$ & $1,816.0$ & $1,825.0$ & $1,780.9$ & $1,837.6$ \\
\hline
\end{tabular}

Sources: Ministry of Finance, and Fund Staff estimates.

1/ Data are for fiscal years beginning April 1. 
Table 11. St. Lucia: Operations of the Central Government 1/

(In million of Eastern Caribbean dollars)

\begin{tabular}{|c|c|c|c|c|c|}
\hline & 1998/99 & 1999/00 & $2000 / 01$ & $2001 / 02$ & $2002 / 03$ \\
\hline Total revenue and grants & 497.8 & 529.9 & 493.6 & 457.9 & 478.1 \\
\hline Current revenue & 436.4 & 465.4 & 482.7 & 428.9 & 441.8 \\
\hline Tax revenue & 402.1 & 418.7 & 433.6 & 384.9 & 405.6 \\
\hline Taxes on income and profits & 105.4 & 127.0 & 138.8 & 118.4 & 105.5 \\
\hline Taxes on property & 1.0 & 0.8 & 1.6 & 4.4 & 4.5 \\
\hline Taxes on goods and services & 189.4 & 170.6 & 151.0 & 152.8 & 174.9 \\
\hline Consumption taxes & 140.7 & 122.8 & 100.8 & 105.4 & 112.7 \\
\hline Imports & 128.2 & 112.2 & 90.3 & 100.1 & 107.8 \\
\hline Domestic goods & 12.5 & 10.6 & 10.5 & 5.3 & 4.9 \\
\hline Hotel occupancy tax & 17.2 & 18.0 & 22.9 & 18.5 & 19.5 \\
\hline Excise duty & 1.1 & 6.3 & 4.5 & 5.5 & 6.4 \\
\hline Other & 25.8 & 23.5 & 22.8 & 23.5 & 36.3 \\
\hline $\begin{array}{l}\text { Taxes on international trade and transactions } \\
\text { Of which }\end{array}$ & 106.3 & 120.3 & 142.2 & 109.3 & 120.8 \\
\hline Import duties & 68.1 & 71.4 & 64.2 & 51.7 & 57.4 \\
\hline Service charge (imports) & 32.8 & 34.7 & 33.4 & 27.9 & 30.2 \\
\hline Nontax revenue & 34.3 & 46.7 & 49.1 & 43.9 & 36.2 \\
\hline Capital revenue & 5.1 & 2.4 & 0.1 & 2.7 & 0.0 \\
\hline Capital grants & 56.2 & 62.1 & 10.8 & 26.3 & 36.3 \\
\hline Total expenditure and net lending & 467.5 & 521.0 & 517.3 & 535.8 & 615.6 \\
\hline Current expenditure & 332.6 & 354.5 & 383.2 & 401.2 & 436.0 \\
\hline Wages and salaries & 182.0 & 198.3 & 209.5 & 212.7 & 211.6 \\
\hline NIS contributions and retirement & 22.9 & 27.4 & 35.6 & 34.3 & 35.2 \\
\hline Goods and services & 66.6 & 71.0 & 74.0 & 72.8 & 75.6 \\
\hline Interest & 21.5 & 23.6 & 29.5 & 42.7 & 45.4 \\
\hline Domestic & 14.3 & 15.8 & 21.3 & 22.5 & 21.9 \\
\hline Foreign & 7.2 & 7.9 & 8.2 & 20.1 & 23.5 \\
\hline Transfers & 39.6 & 34.1 & 34.6 & 38.7 & 68.2 \\
\hline Capital expenditure and net lending & 134.9 & 166.5 & 134.1 & 134.6 & 179.6 \\
\hline Current balance & 103.9 & 110.9 & 99.5 & 27.7 & 5.8 \\
\hline Overall balance before grants & -26.0 & -53.1 & -34.5 & -104.2 & -173.8 \\
\hline Overall balance & 30.2 & 8.9 & -23.7 & -77.9 & -137.5 \\
\hline
\end{tabular}

Sources: Ministry of Finance and ECCB.

1/ Data are for fiscal years beginning April 1. 
Table 12. St. Lucia: Consolidated Accounts of the Nonfinancial Public Enterprises

(In percent of GDP)

\begin{tabular}{|c|c|c|c|c|c|}
\hline & $1998 / 99$ & 1999/00 & $2000 / 01$ & $2001 / 02$ & $\begin{array}{r}\text { Est. } \\
2002 / 03\end{array}$ \\
\hline Total revenue and grants & 4.8 & 5.8 & 5.6 & 5.5 & 5.2 \\
\hline Current revenue & 4.7 & 5.2 & 5.4 & 5.4 & 5.2 \\
\hline Capital revenue & 0.1 & 0.1 & 0.2 & 0.1 & 0.0 \\
\hline Current transfers from Central Government & 0.0 & 0.5 & 0.0 & 0.0 & 0.0 \\
\hline Capital transfers from Central Government & 0.0 & 0.0 & 0.0 & 0.0 & 0.0 \\
\hline Capital grants from abroad & 0.0 & 0.0 & 0.0 & 0.0 & 0.0 \\
\hline Total expenditure & 4.8 & 6.3 & 5.6 & 4.9 & 5.0 \\
\hline Current expenditure & 4.2 & 4.1 & 3.9 & 4.1 & 4.4 \\
\hline \multicolumn{6}{|l|}{ Of which } \\
\hline Interest & 0.5 & 0.6 & 0.6 & 0.6 & 0.5 \\
\hline Capital expenditure & 0.5 & 2.2 & 1.7 & 0.8 & 0.7 \\
\hline Operational balance & 1.0 & 1.7 & 2.1 & 1.9 & 1.3 \\
\hline Current balance & 0.5 & 1.6 & 1.2 & 1.3 & 0.8 \\
\hline Overall balance & 0.0 & -0.5 & 0.0 & 0.5 & 0.2 \\
\hline \multicolumn{6}{|c|}{ Air and Sea Ports Authority 1/ } \\
\hline Total revenue and grants & 2.9 & 3.1 & 2.8 & 2.7 & 2.5 \\
\hline Total expenditure & 2.5 & 3.6 & 3.0 & 2.5 & 3.0 \\
\hline Operational balance & 1.2 & 1.6 & 1.3 & 1.1 & 0.4 \\
\hline Current balance & 0.9 & 1.3 & 1.0 & 0.8 & 0.2 \\
\hline Overall balance & 0.4 & -0.6 & -0.2 & 0.2 & -0.5 \\
\hline \multicolumn{6}{|c|}{ Water and Sewerage Authority 2/ } \\
\hline Total revenue and grants & 1.1 & 2.0 & 1.9 & 2.0 & 2.0 \\
\hline Total expenditure & 1.5 & 1.8 & 1.8 & 1.9 & 1.8 \\
\hline Operational balance & -0.2 & 0.1 & 0.7 & 0.6 & 0.7 \\
\hline Current balance & -0.4 & 0.4 & 0.5 & 0.4 & 0.5 \\
\hline Overall balance & -0.4 & 0.3 & 0.1 & 0.1 & 0.2 \\
\hline \multicolumn{6}{|c|}{ Marketing Board 2/ } \\
\hline Total revenue and grants & 0.3 & 0.2 & 0.2 & 0.3 & 0.2 \\
\hline Total expenditure & 0.3 & 0.2 & 0.2 & 0.2 & 0.2 \\
\hline Operational balance & 0.0 & 0.0 & 0.0 & 0.0 & 0.0 \\
\hline Current balance & 0.0 & 0.0 & 0.0 & 0.0 & 0.0 \\
\hline Overall balance & 0.0 & 0.0 & 0.0 & 0.0 & 0.0 \\
\hline \multicolumn{6}{|c|}{ National Development Corporation 1/ } \\
\hline Total revenue and grants & 0.5 & 0.5 & 0.6 & 0.5 & 0.5 \\
\hline Total expenditure & 0.4 & 0.7 & 0.6 & 0.4 & 0.0 \\
\hline Operational balance & 0.0 & 0.1 & 0.0 & 0.1 & 0.2 \\
\hline Current balance & -0.1 & 0.0 & 0.0 & 0.1 & 0.1 \\
\hline Overall balance & 0.0 & -0.2 & 0.0 & 0.2 & 0.5 \\
\hline
\end{tabular}

Sources: Individual public enterprises; and Fund staff estimates.

1/ Fiscal year begining April 1.

2/ Fiscal year begining January 1. 
Table 13. St. Lucia: Expenditure and Financing of the Public Sector Investment Program (PSIP) 1/

(In percent of GDP)

\begin{tabular}{|c|c|c|c|c|c|}
\hline & 1998/99 & $1999 / 00$ & $2000 / 01$ & $2001 / 02$ & $\begin{array}{r}\text { Est. } \\
2002 / 03 \\
\end{array}$ \\
\hline Total & 7.0 & 8.2 & 6.3 & 7.2 & 8.8 \\
\hline Administration & 0.7 & 1.7 & 0.7 & 0.9 & 1.0 \\
\hline Agriculture & 1.3 & 1.4 & 0.3 & 0.4 & 1.7 \\
\hline Industry & 0.1 & 0.1 & 0.1 & 0.2 & 0.1 \\
\hline Energy 1/ & 0.0 & 0.0 & 0.0 & 0.0 & 0.0 \\
\hline Tourism & 0.8 & 0.7 & 1.1 & 0.9 & 1.1 \\
\hline Transportation & 0.4 & 1.4 & 0.5 & 1.1 & 1.6 \\
\hline Public safety & 0.0 & 0.0 & 0.9 & 0.8 & 0.6 \\
\hline Environment & 0.7 & 1.0 & 0.7 & 1.1 & 0.9 \\
\hline Financial services & 0.0 & 0.0 & 0.0 & 0.0 & 0.0 \\
\hline Education & 0.4 & 0.9 & 1.0 & 0.7 & 0.9 \\
\hline Housing & 0.3 & 0.8 & 0.7 & 0.6 & 0.6 \\
\hline Health & 1.8 & 0.1 & 0.2 & 0.1 & 0.1 \\
\hline Water and sewerage & 0.5 & 0.0 & 0.0 & 0.5 & 0.3 \\
\hline Other & 0.0 & 0.0 & 0.0 & 0.0 & 0.0 \\
\hline Source of financing $1 /$ & 7.0 & 8.2 & 6.3 & 7.2 & 8.8 \\
\hline Domestic & 2.0 & 5.1 & 3.8 & 5.0 & 4.6 \\
\hline External & 5.0 & 3.2 & 2.6 & 2.2 & 4.3 \\
\hline Loans & 4.3 & 4.1 & 0.0 & 0.0 & 0.0 \\
\hline Grants & 1.3 & 1.3 & 0.0 & 0.0 & 0.0 \\
\hline \multicolumn{6}{|l|}{ Memorandum item: } \\
\hline Nominal GDP (EC\$ dollars) & $1,742.4$ & $1,816.0$ & $1,825.0$ & $1,780.9$ & $1,837.6$ \\
\hline
\end{tabular}

Source: Table 21.

1/ Includes investments recorded directly by LUCELEC, not considered in the PSIP of the Ministry of Planning. 
Table 14. St. Lucia: Expenditure and Financing of the Public Sector Investment Program (PSIP)

\begin{tabular}{|c|c|c|c|c|c|}
\hline & $1998 / 99$ & 1999/00 & $2000 / 01$ & $2001 / 02$ & $\begin{array}{r}\text { Est. } \\
2002 / 03\end{array}$ \\
\hline \multicolumn{6}{|c|}{ (In millions of Eastern Caribbean dollars) } \\
\hline Total & 121.4 & 149.5 & 115.8 & 129.0 & 162.6 \\
\hline General public service & 12.4 & 31.8 & 29.1 & 30.2 & 30.3 \\
\hline Administration & 11.9 & 31.6 & 13.0 & 16.1 & 19.1 \\
\hline Public safety & 0.5 & 0.3 & 16.1 & 14.1 & 11.2 \\
\hline Economic sectors & 38.7 & 40.2 & 27.6 & 26.9 & 53.6 \\
\hline Agriculture & 22.6 & 25.0 & 5.9 & 7.2 & 31.6 \\
\hline Trade and industry & 2.4 & 2.1 & 1.2 & 3.2 & 1.5 \\
\hline Tourism & 13.7 & 13.1 & 20.5 & 16.5 & 20.5 \\
\hline Economic infrastructure & 14.9 & 26.1 & 10.8 & 27.7 & 34.8 \\
\hline Power & 0.0 & 0.6 & 0.5 & 0.2 & 0.2 \\
\hline Transport and communication & 7.0 & 25.3 & 9.4 & 18.9 & 29.0 \\
\hline Water and sanitation & 7.9 & 0.2 & 0.9 & 8.6 & 5.5 \\
\hline Social sectors & 43.3 & 33.7 & 34.8 & 25.1 & 28.1 \\
\hline Education & 7.7 & 16.5 & 17.6 & 12.9 & 16.5 \\
\hline Housing and community services & 4.7 & 14.9 & 12.7 & 10.1 & 10.4 \\
\hline Health & 30.9 & 2.3 & 4.5 & 2.1 & 1.1 \\
\hline Other & 12.1 & 17.8 & 13.5 & 19.1 & 15.9 \\
\hline Environment & 12.1 & 17.8 & 13.5 & 19.1 & 15.8 \\
\hline Financial services & 0.0 & 0.0 & 0.0 & 0.0 & 0.1 \\
\hline Other & 0.0 & 0.0 & 0.0 & 0.0 & 0.0 \\
\hline Source of financing & 121.4 & 149.5 & 115.8 & 129.1 & 162.5 \\
\hline Domestic & 34.0 & 91.8 & 69.2 & 89.9 & 84.0 \\
\hline External & 87.4 & 57.7 & 46.6 & 39.2 & 78.5 \\
\hline Loans & 75.2 & 75.2 & $\ldots$ & $\ldots$ & $\ldots$ \\
\hline Grants & 23.5 & 23.5 & $\ldots$ & $\ldots$ & $\ldots$ \\
\hline \multicolumn{6}{|c|}{ (In percent of public sector investment) } \\
\hline Total & 100.0 & 100.0 & 100.0 & 100.0 & 100.0 \\
\hline General public service & 10.2 & 21.3 & 25.1 & 23.4 & 18.6 \\
\hline Administration & 9.8 & 21.1 & 11.2 & 12.5 & 11.7 \\
\hline Public safety & 0.4 & 0.2 & 13.9 & 10.9 & 6.9 \\
\hline Economic sectors & 31.9 & 26.9 & 23.8 & 20.9 & 33.0 \\
\hline Agriculture & 18.6 & 16.7 & 5.1 & 5.6 & 19.4 \\
\hline Trade and industry & 2.0 & 1.4 & 1.0 & 2.5 & 0.9 \\
\hline Tourism & 11.3 & 8.8 & 17.7 & 12.8 & 12.6 \\
\hline Economic infrastructure & 12.3 & 17.4 & 9.3 & 21.5 & 21.4 \\
\hline Power & 0.0 & 0.4 & 0.4 & 0.2 & 0.1 \\
\hline Transport and communication & 5.8 & 16.9 & 8.1 & 14.7 & 17.9 \\
\hline Water and sanitation & 6.5 & 0.1 & 0.8 & 6.7 & 3.4 \\
\hline Social sectors & 35.7 & 22.5 & 30.1 & 19.5 & $\mathbf{1 7 . 3}$ \\
\hline Education & 6.3 & 11.0 & 15.2 & 10.0 & 10.2 \\
\hline Housing and community services & 3.9 & 9.9 & 11.0 & 7.8 & 6.4 \\
\hline Health & 25.5 & 1.5 & 3.9 & 1.6 & 0.7 \\
\hline Other & 10.0 & 11.9 & 11.7 & 14.8 & 9.8 \\
\hline Environment & 10.0 & 11.9 & 11.7 & 14.8 & 9.7 \\
\hline Financial services & 0.0 & 0.0 & 0.0 & 0.0 & 0.0 \\
\hline Other & 0.0 & 0.0 & 0.0 & 0.0 & 0.0 \\
\hline Source of financing & 100.0 & 100.0 & 100.0 & 100.0 & 100.0 \\
\hline Domestic & 28.0 & 61.4 & 59.8 & 69.7 & 51.7 \\
\hline External & 72.0 & 38.6 & 40.2 & 30.4 & 48.3 \\
\hline Loans & 61.9 & 50.3 & $\ldots$ & $\ldots$ & $\ldots$ \\
\hline Grants & 19.4 & 15.7 & $\ldots$ & $\ldots$ & $\ldots$ \\
\hline
\end{tabular}

Source: Ministry of Planning; and Fund staff estimates. 
Table 15. St. Lucia: Monetary Survey

\begin{tabular}{|c|c|c|c|c|c|}
\hline & 1999 & 2000 & 2001 & 2002 & $\begin{array}{r}\text { Est } \\
2003 \\
\end{array}$ \\
\hline \multicolumn{6}{|c|}{ (In millions of Eastern Caribbean dollars, end of period) } \\
\hline Net foreign assets & 81.4 & 116.6 & 117.4 & 105.2 & 246.4 \\
\hline Net imputed international reserves & 195.6 & 207.8 & 235.2 & 248.1 & 294.5 \\
\hline Commercial banks & -114.2 & -91.2 & -117.8 & -142.8 & -48.1 \\
\hline Net domestic assets & 1,011.9 & $1,062.8$ & $1,116.9$ & $1,168.1$ & $1,087.9$ \\
\hline Net credit to the public sector & -239.1 & -258.4 & -281.6 & -250.7 & -282.9 \\
\hline Claims on government (net) & -63.8 & -85.8 & -113.1 & -96.3 & -97.4 \\
\hline ECCB net credit to central government & 7.9 & 3.5 & 10.9 & 3.1 & -7.5 \\
\hline Commercial bank net credit to government & -71.7 & -89.3 & -124.0 & -99.4 & -89.9 \\
\hline Net credit to other public sector & -175.4 & -172.7 & -168.6 & -154.3 & -185.5 \\
\hline Credit to private sector $1 /$ & $1,394.2$ & $1,481.7$ & $1,541.8$ & $1,553.5$ & $1,500.8$ \\
\hline Other items (net) & -143.2 & -160.5 & -143.3 & -134.7 & -130.0 \\
\hline Money and quasi-money (M2) & $1,093.3$ & $1,179.4$ & $1,234.3$ & $1,273.3$ & $1,334.3$ \\
\hline Money & 267.6 & 286.1 & 283.8 & 287.6 & 304.9 \\
\hline Currency outside banks & 84.1 & 84.6 & 82.1 & 83.6 & 84.2 \\
\hline Demand deposits & 183.6 & 201.5 & 201.7 & 204.0 & 222.3 \\
\hline Quasi-money & 825.7 & 893.3 & 950.5 & 985.7 & $1,029.4$ \\
\hline Time deposits & 292.2 & 334.7 & 364.4 & 309.6 & 255.5 \\
\hline Savings deposits & 512.5 & 551.1 & 573.3 & 652.3 & 743.0 \\
\hline Foreign currency deposits & 20.9 & 7.6 & 12.8 & 23.8 & 30.9 \\
\hline \multicolumn{6}{|c|}{ (12-month change in percent of M2 at the beginning of the period) } \\
\hline Net foreign assets & -1.1 & 3.2 & 0.1 & -1.0 & 11.1 \\
\hline Net imputed reserves & 1.1 & 1.1 & 2.3 & 1.0 & 3.6 \\
\hline Commercial banks & -2.2 & 2.1 & -2.3 & -2.0 & 7.4 \\
\hline Net domestic assets & 10.8 & 4.7 & 4.6 & 4.1 & -6.3 \\
\hline Credit to the public sector & -2.9 & -1.8 & -2.0 & 2.5 & -2.5 \\
\hline Credit to the central government & -2.5 & -2.0 & -2.3 & 1.4 & -0.1 \\
\hline Credit to the rest of the public sector & -0.4 & 0.2 & 0.3 & 1.2 & -2.4 \\
\hline Credit to the private sector & 13.6 & 8.0 & 5.1 & 0.9 & -4.1 \\
\hline Other items (net) & 0.1 & -1.6 & 1.5 & 0.7 & 0.4 \\
\hline \multicolumn{6}{|c|}{ (12-month percentage change) } \\
\hline Credit to the private sector & 10.8 & 6.3 & 4.1 & 0.8 & -3.4 \\
\hline Money and quasi-money & 9.7 & 7.9 & 4.7 & 3.2 & 4.8 \\
\hline Money & 8.0 & 6.9 & -0.8 & 1.3 & 6.0 \\
\hline Quasi-money & 10.3 & 8.2 & 6.4 & 3.7 & 4.4 \\
\hline \multicolumn{6}{|l|}{ Memorandum item: } \\
\hline Income velocity of M2 2/ & 1.7 & 1.6 & 1.5 & 1.5 & 1.4 \\
\hline
\end{tabular}

Sources: ECCB; and Fund staff estimates and projections.

1/ Includes assumption by government of EC $\$ 40$ million in debt from the St. Lucia Banana Growers Association in 1998, previously included under credit to the private sector.

2/ Nominal GDP at market prices divided by the average stock of money (measured as the simple average of the current stpekiadd the stock 12-months earlier). 
Table 16. St. Lucia: Summary Operations of the Eastern Caribbean Central Bank (In millions of Eastern Caribbean dollars)

\begin{tabular}{|c|c|c|c|c|c|}
\hline & 1998 & 1999 & 2000 & 2001 & 2002 \\
\hline Net (imputed) international reserves 1 / & 185.0 & 195.6 & 207.8 & 235.2 & 248.1 \\
\hline Net claims on commercial banks & -118.4 & -117.5 & -125.4 & -164.0 & -167.6 \\
\hline Claims & 0.1 & 0.1 & 0.0 & 0.1 & 0.0 \\
\hline Liabilities & 118.5 & 117.5 & 125.5 & 164.0 & 167.6 \\
\hline Currency held by banks & 43.8 & 40.4 & 37.3 & 37.0 & 40.8 \\
\hline Statutory reserves & 71.0 & 73.4 & 84.4 & 123.3 & 126.2 \\
\hline Fixed deposits & 3.8 & 3.8 & 3.8 & 3.8 & 0.7 \\
\hline Net claims on statutory bodies & -1.3 & -2.0 & -1.2 & 0.0 & $\mathbf{0 . 0}$ \\
\hline Net domestic assets & 12.2 & 7.9 & 3.5 & 10.9 & 3.1 \\
\hline Net credit to central government & 12.2 & 7.9 & 3.5 & 10.9 & 3.1 \\
\hline Short-term credit & 0.0 & 0.0 & 0.0 & 0.0 & 0.0 \\
\hline Temporary advances & 1.7 & 1.2 & 1.9 & 1.9 & 3.3 \\
\hline Treasury bills & 5.2 & 6.6 & 5.4 & 0.8 & 0.2 \\
\hline Debentures & 5.0 & 5.0 & 5.0 & 5.0 & 5.0 \\
\hline Other claims & 6.5 & 5.4 & 4.3 & 3.2 & 2.2 \\
\hline Central government deposits & -6.2 & -10.3 & -13.1 & 0.0 & -7.7 \\
\hline Currency in circulation & 77.5 & 84.1 & 84.6 & 82.1 & 83.6 \\
\hline Total currency issued & 121.3 & 124.4 & 121.9 & 119.1 & 124.4 \\
\hline Less: Currency held by banks & 43.8 & 40.4 & 37.3 & 37.0 & 40.8 \\
\hline
\end{tabular}

Source: Eastern Caribbean Central Bank.

1/ Share of St. Lucia in the international reserves of the ECCB, calculated as the difference between liabilities to the private sector and net claims on commercial banks and government. 
Table 17. St. Lucia: Consolidated Accounts of the Commercial Banks 1/ (In millions of Eastern Caribbean dollars)

\begin{tabular}{|c|c|c|c|c|c|}
\hline & 1998 & 1999 & 2000 & 2001 & 2002 \\
\hline Net foreign assets & -92.4 & -114.2 & -91.2 & -117.8 & -142.8 \\
\hline Foreign assets & 143.2 & 146.5 & 136.0 & 176.1 & 228.1 \\
\hline Foreign currency holdings & 9.8 & 7.4 & 7.3 & 7.9 & 10.5 \\
\hline Claims on ECCB area banks & 48.7 & 37.5 & 24.4 & 56.2 & 78.9 \\
\hline Claims on banks abroad & 31.9 & 28.2 & 34.4 & 47.9 & 47.1 \\
\hline Other $2 /$ & 52.9 & 73.4 & 69.9 & 64.1 & 91.6 \\
\hline Foreign liabilities & 235.7 & 260.8 & 227.2 & 293.8 & 370.9 \\
\hline Balances due to ECCB area banks & 44.4 & 40.4 & 43.2 & 20.8 & 49.4 \\
\hline Balances due to banks abroad & 69.6 & 97.5 & 64.8 & 62.0 & 94.7 \\
\hline Nonresident deposits & 121.0 & 122.5 & 118.7 & 131.3 & 147.2 \\
\hline Other & 0.7 & 0.5 & 0.4 & 79.8 & 79.7 \\
\hline Net position with ECCB & 108.5 & 123.1 & 120.1 & 150.3 & 172.8 \\
\hline Net claims on ECCB & 108.5 & 123.5 & 121.0 & 152.4 & 173.9 \\
\hline Currency holdings & 43.8 & 40.4 & 37.3 & 37.0 & 40.8 \\
\hline Current deposits & 61.0 & 79.4 & 74.6 & 111.7 & 112.4 \\
\hline Fixed deposits and call accounts & 3.8 & 3.8 & 9.1 & 3.8 & 20.7 \\
\hline Liabilities to ECCB & 0.0 & -0.5 & -0.9 & -2.1 & -1.0 \\
\hline Net domestic assets & 902.8 & $1,000.4$ & $1,065.9$ & $1,121.2$ & $1,159.7$ \\
\hline Net credit to government & -51.1 & -71.7 & -89.3 & -124.0 & -99.4 \\
\hline Treasury bills & 11.2 & 11.2 & 10.2 & 18.3 & 20.3 \\
\hline Other securities & 15.5 & 23.5 & 34.6 & 23.3 & 52.2 \\
\hline Loans and advances 3 / & 85.2 & 87.0 & 85.9 & 87.7 & 70.1 \\
\hline Deposits & -163.0 & -193.4 & -219.9 & -253.3 & -242.1 \\
\hline Net credit to National Insurance Scheme & -156.4 & -163.0 & -184.1 & -183.2 & -174.3 \\
\hline Net credit to public enterprises & -14.9 & -12.4 & 11.5 & 14.7 & 20.0 \\
\hline Loans, advances, and investments & 46.7 & 47.1 & 65.5 & 64.6 & 69.8 \\
\hline Deposits & -61.6 & -59.4 & -54.1 & -49.9 & -49.8 \\
\hline Net credit to other financial institutions & -49.9 & -38.6 & -24.9 & -43.4 & -32.7 \\
\hline Loans, advances, and investments & 14.1 & 30.5 & 30.7 & 25.9 & 28.3 \\
\hline Deposits & -64.1 & -69.1 & -55.6 & -69.3 & -61.0 \\
\hline Credit to private sector & $1,258.2$ & $1,394.2$ & $1,481.7$ & $1,541.8$ & $1,553.5$ \\
\hline Interbank float & 9.8 & 27.1 & 14.1 & 7.8 & 1.7 \\
\hline Claims & 48.2 & 70.2 & 41.2 & 37.6 & 28.1 \\
\hline Liabilities & -38.4 & -43.1 & -27.1 & -29.8 & -26.5 \\
\hline Net unclassified assets & -92.8 & -135.2 & -143.1 & -92.6 & -108.9 \\
\hline Liabilities to the private sector 4 / & 918.9 & $1,009.2$ & $1,094.8$ & $1,152.2$ & $1,189.7$ \\
\hline Demand deposits & 170.4 & 183.6 & 201.5 & 201.7 & 204.0 \\
\hline Saving deposits & 467.9 & 512.5 & 551.1 & 573.3 & 652.3 \\
\hline Time deposits & 273.6 & 292.2 & 334.7 & 364.4 & 309.6 \\
\hline Foreign currency deposits & 7.1 & 20.9 & 7.6 & 12.8 & 23.8 \\
\hline
\end{tabular}

Sources: Eastern Caribbean Central Bank; and Fund staff projections.

1/ The National Commercial Bank, the St. Lucia Cooperative Bank, Barclays Bank, the Bank of Nova Scotia, the Canadian Imperial Bank of Commerce, the Royal Bank of Canada, and Caribbean Banking Corporation.

2/ Includes financial instrument issued by and loans to nonbank residents of countries ouside ECCB territory.

3/ Includes government assumption of EC \$40 million in debt from the SLBGA in 1998, previously included under credit to the private sector.

4/ Private sector includes nonbank financial institutions. 
Table 18. St. Lucia: Selected Interest Rates

\begin{tabular}{lllllll}
\hline & \multicolumn{3}{c}{ December 31 } & & September 30 \\
\cline { 2 - 4 } \cline { 5 - 6 } & 1999 & 2000 & 2001 & & 2002 & 2003 \\
\hline
\end{tabular}

I. Eastern Caribbean Central Bank

Deposit Rates for Commercial Banks

(In percent per annum)

\section{Call}

One month

Three months

\section{Lending}

Overdrafts

Temporary advances

$\begin{array}{llll}5.36 & 6.38 & 1.79 & 1.66 \\ 6.09 & 6.46 & 1.67 & 1.60 \\ 5.91 & 6.23 & 1.65 & 1.58\end{array}$

$\begin{array}{rrrr}10.50 & 10.50 & 10.50 & 10.50 \\ 8.00 & 8.00 & 7.00 & 7.00\end{array}$

Memorandum items:

Libor on U.S. dollar three-month deposits (average)

$$
5.41
$$

3.78

1.81

Difference between Libor and comparable ECCB rate

$\begin{array}{llll}0.50 & -0.31 & -2.13 & -0.23\end{array}$

II. Commercial Banks

\section{Deposits}

Savings

Time

$\begin{array}{llllll}3 \text { months } & 2.00-9.25 & 2.00-9.25 & 2.00-6.000 & 2.00-6.00 & 2.00-6.00 \\ 6 \text { months } & 2.00-9.25 & 2.00-9.25 & 2.00-6.250 & 2.00-6.00 & 2.00-6.25 \\ 12 \text { months } & 2.00-9.25 & 2.00-9.25 & 2.00-6.500 & 2.00-6.00 & 2.00-6.50\end{array}$

\section{Lending}

Prime rate

$$
\begin{array}{rrrrr}
9.50-10.50 & 9.50-10.50 & 9.50-10.500 & 9.50-10.50 & 9.50-10.50 \\
7.5-18.00 & 7.5-18.00 & 6.00-18.000 & 6.00-18.00 & 6.00-18.00
\end{array}
$$

Sources: Ministry of Finance; and Eastern Caribbean Central Bank. 
Table 19. St. Lucia: Sectoral Distribution of Commercial Bank Loans and Advances

\begin{tabular}{|c|c|c|c|c|c|}
\hline & 1998 & 1999 & 2000 & 2001 & 2002 \\
\hline \multicolumn{6}{|c|}{ (In millions of Eastern Caribbean dollars at end of period) } \\
\hline Total & $1,422.2$ & $1,560.5$ & $1,649.5$ & $1,709.2$ & $1,721.2$ \\
\hline Public sector & 127.0 & 131.2 & 144.5 & 156.8 & 149.9 \\
\hline Government and public enterprises, n.i.e. & 108.5 & 115.2 & 124.2 & 125.5 & 112.3 \\
\hline Public utilities & 18.6 & 15.9 & 20.2 & 31.4 & 37.5 \\
\hline Businesses & 592.0 & 687.7 & 703.9 & 759.3 & 813.2 \\
\hline Agriculture and fisheries & 21.8 & 23.7 & 30.0 & 41.1 & 34.9 \\
\hline Manufacturing and mining & 53.4 & 53.0 & 46.5 & 53.8 & 56.9 \\
\hline Distributive trades & 178.6 & 195.4 & 200.3 & 198.9 & 214.0 \\
\hline Tourism and entertainment & 133.8 & 163.1 & 155.2 & 172.6 & 212.2 \\
\hline Transport & 39.5 & 48.2 & 55.8 & 49.0 & 33.2 \\
\hline Construction and land development & 59.3 & 66.5 & 84.5 & 99.9 & 110.9 \\
\hline Professional and other services & 93.7 & 125.8 & 119.0 & 134.4 & 135.9 \\
\hline Financial institutions & 12.0 & 12.1 & 12.5 & 9.5 & 15.2 \\
\hline Households & 703.1 & 741.6 & 801.2 & 793.1 & 758.1 \\
\hline Acquisition of property & 303.3 & 337.7 & 378.3 & 319.5 & 325.7 \\
\hline Durable consumer goods & 127.7 & 99.4 & 103.8 & 90.1 & 63.9 \\
\hline Other & 272.0 & 304.5 & 319.1 & 383.5 & 368.6 \\
\hline \multicolumn{6}{|c|}{ (In percent of total) } \\
\hline Public sector & 8.9 & 8.4 & 8.8 & 9.2 & 8.7 \\
\hline Government and public enterprises, n.i.e. & 7.6 & 7.4 & 7.5 & 7.3 & 6.5 \\
\hline Public utilities & 1.3 & 1.0 & 1.2 & 1.8 & 2.2 \\
\hline Businesses & 41.6 & 44.1 & 42.7 & 44.4 & 47.2 \\
\hline Agriculture and fisheries & 1.5 & 1.5 & 1.8 & 2.4 & 2.0 \\
\hline Manufacturing and mining & 3.8 & 3.4 & 2.8 & 3.1 & 3.3 \\
\hline Distributive trades & 12.6 & 12.5 & 12.1 & 11.6 & 12.4 \\
\hline Tourism and entertainment & 9.4 & 10.5 & 9.4 & 10.1 & 12.3 \\
\hline Transport & 2.8 & 3.1 & 3.4 & 2.9 & 1.9 \\
\hline Construction and land development & 4.2 & 4.3 & 5.1 & 5.8 & 6.4 \\
\hline Professional and other services & 6.6 & 8.1 & 7.2 & 7.9 & 7.9 \\
\hline Financial institutions & 0.8 & 0.8 & 0.8 & 0.6 & 0.9 \\
\hline Households & 49.4 & 47.5 & 48.6 & 46.4 & 44.0 \\
\hline House and land purchases & 21.3 & 21.6 & 22.9 & 18.7 & 18.9 \\
\hline Durable consumer goods & 9.0 & 6.4 & 6.3 & 5.3 & 3.7 \\
\hline Other & 19.1 & 19.5 & 19.3 & 22.4 & 21.4 \\
\hline
\end{tabular}

Source: Eastern Caribbean Central Bank. 
Table 20. St. Lucia: Summary Balance of Payments

\begin{tabular}{|c|c|c|c|c|c|}
\hline & 1999 & 2000 & 2001 & 2002 & $\begin{array}{r}\text { Est. } \\
2003\end{array}$ \\
\hline \multicolumn{6}{|c|}{ (In millions of U.S. dollars) } \\
\hline Current account & -83.6 & -84.8 & -59.0 & -84.9 & -129.1 \\
\hline Trade balance & -251.1 & -259.6 & -218.0 & -205.7 & -286.4 \\
\hline Exports, f.o.b. & 60.9 & 52.9 & 54.1 & 69.8 & 66.1 \\
\hline Imports, f.o.b. & -312.0 & -312.5 & -272.1 & -275.6 & -352.6 \\
\hline Services (net) & 145.5 & 157.7 & 145.4 & 107.9 & 143.3 \\
\hline Receipts & 322.7 & 332.5 & 301.7 & 278.4 & 322.5 \\
\hline Travel & 277.1 & 286.1 & 251.2 & 230.7 & 266.7 \\
\hline Other nonfactor services & 43.2 & 42.5 & 47.9 & 45.1 & 53.2 \\
\hline Factor services & 2.4 & 3.9 & 2.6 & 2.6 & 2.6 \\
\hline Payments & 177.2 & 174.8 & 156.3 & 170.5 & 179.2 \\
\hline Travel & 33.0 & 33.3 & 31.5 & 33.5 & 34.3 \\
\hline Other nonfactor services & 103.4 & 94.5 & 79.6 & 91.1 & 93.2 \\
\hline Factor services & 40.8 & 47.0 & 45.2 & 46.0 & 51.6 \\
\hline Of which: public interest payments & 6.9 & 8.1 & 11.5 & 10.7 & 15.5 \\
\hline Current transfers (net) & 22.0 & 17.1 & 13.6 & 13.0 & 13.9 \\
\hline Capital and Financial Account & 91.3 & 93.0 & 69.2 & 90.0 & 146.3 \\
\hline Capital transfers (net) & 25.1 & 15.8 & 26.4 & 21.4 & 25.9 \\
\hline Official borrowing (net) & 13.3 & 29.5 & 20.9 & 41.5 & 67.5 \\
\hline Central government loan drawings & 23.3 & 17.9 & 32.6 & 31.0 & 20.4 \\
\hline Central government loan amortization & 10.0 & 12.0 & 25.1 & 23.5 & 6.5 \\
\hline Other (net) & 0.0 & 23.5 & 13.4 & 33.9 & 53.6 \\
\hline Private capital & 52.9 & 47.8 & 21.8 & 27.1 & 52.9 \\
\hline Private long-term (net) & 43.3 & 45.1 & 10.8 & 15.2 & 84.5 \\
\hline Of which: direct investment (net) & 82.8 & 54.9 & 22.4 & 31.4 & 102.1 \\
\hline Commercial banks & 8.1 & -8.5 & 9.8 & 7.6 & -35.1 \\
\hline Other short term capital (net) $1 /$ & 1.6 & 11.2 & 1.2 & 4.3 & 3.5 \\
\hline Overall balance & 7.7 & 8.2 & 10.1 & 5.1 & $\mathbf{1 7 . 2}$ \\
\hline Financing (increase -) & -7.7 & -8.2 & -10.1 & -5.1 & -17.2 \\
\hline Change in SDR holdings & 0.0 & 0.0 & 0.0 & 0.0 & 0.0 \\
\hline Change in government foreign assets & -3.8 & -3.7 & 0.0 & -0.4 & 0.0 \\
\hline Change in imputed reserves (increase -) & -3.9 & -4.5 & -10.1 & -4.8 & -17.2 \\
\hline \multicolumn{6}{|c|}{ (In percent of GDP) } \\
\hline Current account & -12.5 & -12.4 & -9.0 & -12.5 & -18.6 \\
\hline Capital and financial account & 13.6 & 13.6 & 10.6 & 13.3 & 21.1 \\
\hline
\end{tabular}

Sources: St. Lucian authorities; ECCB; and Fund staff estimates.

1/ Includes errors and omissions. 
Table 21. St. Lucia: Value, Volume, and Unit Value of Major Exports

\begin{tabular}{|c|c|c|c|c|c|}
\hline & 1999 & 2000 & 2001 & 2002 & 2003 \\
\hline \multicolumn{6}{|c|}{ (Values in thousands of U.S. dollars and units in U.S. dollars ) } \\
\hline Total goods exports & 60,894 & 52,861 & 54,116 & 69,839 & 66,143 \\
\hline Domestic exports & 51,969 & 39,357 & 37,142 & 42,882 & 37,729 \\
\hline Re-exports & 3,677 & 3,889 & 7,159 & 19,016 & 21,356 \\
\hline Other $1 /$ & 5,248 & 9,615 & 9,815 & 7,941 & 7,058 \\
\hline \multicolumn{6}{|l|}{ Main domestic exports } \\
\hline \multicolumn{6}{|l|}{ Bananas } \\
\hline Value & 32,211 & 31,841 & 15,244 & 21,707 & 15,300 \\
\hline Volume ('000 tons) & 65 & 70 & 34 & 48 & 31 \\
\hline Unit price & 493.82 & 453.04 & 447.78 & 450.72 & 487.26 \\
\hline \multicolumn{6}{|l|}{ Fruits and vegetables } \\
\hline Value & 2,055 & 1,727 & 1,835 & $\ldots$ & $\ldots$ \\
\hline Volume ('000 kilos) & 3,939 & 3,954 & 3,291 & $\ldots$ & $\ldots$ \\
\hline Unit price & 0.52 & 0.44 & 0.56 & $\ldots$ & $\ldots$ \\
\hline \multicolumn{6}{|l|}{ Beer } \\
\hline Value & 6,541 & 7,401 & 9,158 & $\ldots$ & $\ldots$ \\
\hline Volume('000 gallons) & 846 & 979 & 1,333 & $\ldots$ & $\cdots$ \\
\hline Unit price & 7.73 & 7.56 & 6.87 & $\ldots$ & $\ldots$ \\
\hline \multicolumn{6}{|l|}{ Paper and paperboard } \\
\hline Value & 2,420 & 2,472 & 1,170 & 3,770 & $\ldots$ \\
\hline Volume ('000 kilos) & 3,009 & 3,242 & 1,570 & 4,253 & $\ldots$ \\
\hline Unit price & 0.80 & 0.76 & 0.75 & 0.89 & $\ldots$ \\
\hline \multicolumn{6}{|l|}{ Clothing } \\
\hline Value & 3,309 & 3,293 & 3,378 & ... & $\ldots$ \\
\hline Volume (000 units) & 1,920 & 2,016 & 2,117 & $\ldots$ & $\ldots$ \\
\hline Unit price & 1.72 & 1.63 & 1.60 & $\ldots$ & $\cdots$ \\
\hline \multicolumn{6}{|l|}{ Electronic components } \\
\hline Value & 1,832 & 2,272 & 2,331 & $\ldots$ & $\ldots$ \\
\hline Volume (kilos) & 45 & 47 & 50 & $\ldots$ & $\ldots$ \\
\hline Unit price & 40.71 & 48.34 & 46.62 & $\ldots$ & $\ldots$ \\
\hline \multicolumn{6}{|c|}{ (In percent of GDP) } \\
\hline Total Goods Exports & 9.1 & 7.7 & 8.3 & 10.3 & 9.5 \\
\hline Domestic exports & 7.8 & 5.8 & 5.7 & 6.3 & 5.4 \\
\hline Re-exports & 0.5 & 0.6 & 1.1 & 2.8 & 3.1 \\
\hline Other 1/ & 0.8 & 1.4 & 1.5 & 1.2 & 1.0 \\
\hline \multicolumn{6}{|c|}{ (Annual percentage change) } \\
\hline Total Goods Exports & -13.5 & -13.2 & 2.4 & 29.1 & -5.3 \\
\hline Domestic exports & -13.9 & -24.3 & -5.6 & 15.5 & -12.0 \\
\hline Re-exports & 102.7 & 5.8 & 84.1 & 165.6 & 12.3 \\
\hline Other 1/ & -35.8 & 83.2 & 2.1 & -19.1 & -11.1 \\
\hline
\end{tabular}

Sources: Statistics Department; St. Lucia Banana Growers Association, Windward Islands Banana Development and Exporting Company (Wibdeco); and Fund staff estimates.

1/ Includes goods procured in ports by carriers and repair on goods. 
Table 22. St. Lucia: Merchandise Imports by SITC Category

\begin{tabular}{|c|c|c|c|c|c|}
\hline & 1999 & 2000 & 2001 & 2002 & $\begin{array}{r}\text { Est. } \\
2003 \\
\end{array}$ \\
\hline \multicolumn{6}{|c|}{ (In millions of U.S. dollars) } \\
\hline Total imports, c.i.f. & 354.6 & 355.1 & 309.2 & 313.2 & 400.6 \\
\hline Food & 70.8 & 71.0 & 69.0 & 62.6 & 70.9 \\
\hline Beverages and tobacco & 12.1 & 12.1 & 11.8 & 11.7 & 13.1 \\
\hline Crude materials & 10.6 & 10.5 & 8.6 & 8.0 & 8.6 \\
\hline Fuels & 24.1 & 33.2 & 39.6 & 31.0 & 41.6 \\
\hline Oils and fats & 0.6 & 0.6 & 0.6 & 0.3 & 0.7 \\
\hline Chemicals & 32.3 & 29.2 & 26.0 & 26.1 & 28.3 \\
\hline Manufactured goods & 72.1 & 62.8 & 55.7 & 52.3 & 58.6 \\
\hline Machinery and transport equipment & 81.9 & 87.2 & 61.2 & 72.1 & 121.8 \\
\hline Miscellaneous manufactured articles & 49.7 & 48.5 & 36.7 & 49.0 & 57.0 \\
\hline Miscellaneous & 0.3 & 0.0 & 0.0 & 0.1 & 0.1 \\
\hline \multicolumn{6}{|c|}{ (In percent) } \\
\hline Total imports, c.i.f. & 100.0 & 100.0 & 100.0 & 100.0 & 100.0 \\
\hline Food & 20.0 & 20.0 & 22.3 & 20.0 & 17.7 \\
\hline Beverages and tobacco & 3.4 & 3.4 & 3.8 & 3.7 & 3.3 \\
\hline Crude materials & 3.0 & 3.0 & 2.8 & 2.5 & 2.2 \\
\hline Fuels & 6.8 & 9.3 & 12.8 & 9.9 & 10.4 \\
\hline Oils and fats & 0.2 & 0.2 & 0.2 & 0.1 & 0.2 \\
\hline Chemicals & 9.1 & 8.2 & 8.4 & 8.3 & 7.1 \\
\hline Manufactured goods & 20.3 & 17.7 & 18.0 & 16.7 & 14.6 \\
\hline Machinery and transport equipment & 23.1 & 24.6 & 19.8 & 23.0 & 30.4 \\
\hline Miscellaneous manufactured articles & 14.0 & 13.7 & 11.9 & 15.6 & 14.2 \\
\hline Miscellaneous & 0.1 & 0.0 & 0.0 & 0.0 & 0.0 \\
\hline \multicolumn{6}{|l|}{ Memorandum items: } \\
\hline \multicolumn{6}{|l|}{ Total imports, c.i.f. } \\
\hline Percent change & 5.8 & 0.1 & -12.9 & 1.3 & 27.9 \\
\hline Ratio to GDP (percent) & 53.0 & 52.0 & 47.3 & 46.3 & 57.8 \\
\hline
\end{tabular}

Source: Statistics Department of the Ministry of Finance;and Fund staff estimates. 
Table 23. St. Lucia: Imports and Prices of Petroleum Products

(Values in millions of Eastern Caribbean dollar, quantities in millions of gallons, and prices in Eastern Caribbean dollar per gallon; unless otherwise indicated)

\begin{tabular}{|c|c|c|c|c|c|}
\hline & 1999 & 2000 & 2001 & 2002 & $20031 /$ \\
\hline \multicolumn{6}{|l|}{ Gasoline } \\
\hline Value & 25.3 & 39.4 & 38.8 & 37.9 & 21.8 \\
\hline Volume & 14.4 & 11.9 & 12.7 & $\ldots$ & $\ldots$ \\
\hline Unit price & 1.8 & 3.3 & 3.1 & $\ldots$ & $\ldots$ \\
\hline Retail price & 6.4 & 7.0 & 7.0 & 7.8 & 7.8 \\
\hline \multicolumn{6}{|l|}{ Diesel } \\
\hline Value & 27.2 & 12.6 & 10.9 & 31.5 & 42.5 \\
\hline Volume & 15.2 & 4.1 & 3.9 & $\ldots$ & $\ldots$ \\
\hline Unit price & 1.8 & 3.1 & 2.8 & $\ldots$ & $\ldots$ \\
\hline Retail price & 5.9 & 6.5 & 6.5 & 7.0 & 7.0 \\
\hline \multicolumn{6}{|l|}{ Kerosene } \\
\hline Value & 0.2 & 2.5 & 3.2 & 0.4 & 0.4 \\
\hline Volume & 0.1 & 0.8 & 1.1 & $\ldots$ & $\ldots$ \\
\hline Unit price & 2.0 & 3.2 & 2.9 & $\ldots$ & $\ldots$ \\
\hline Retail price & 5.0 & 5.2 & 5.2 & 5.2 & 5.2 \\
\hline \multicolumn{6}{|l|}{ LPG (gas) } \\
\hline Value & 3.3 & $\ldots$ & $\ldots$ & $\ldots$ & $\ldots$ \\
\hline Volume & 2.6 & $\ldots$ & $\ldots$ & $\ldots$ & $\ldots$ \\
\hline Unit price $(\mathrm{EC} \$ / \mathrm{lb})$ & 1.3 & 1.4 & 1.4 & 1.4 & 1.4 \\
\hline \multicolumn{6}{|l|}{ Memorandum items: } \\
\hline Electricity consumption (millions of kwh) & 254.4 & 274.9 & 284.7 & 284.5 & 203.6 \\
\hline \multicolumn{6}{|l|}{ Of which } \\
\hline Domestic & 79.5 & 85.1 & 88.5 & 89.1 & 61.7 \\
\hline Commercial & 79.3 & 85.2 & 88.6 & 86.7 & 60.5 \\
\hline Industrial & 12.3 & 13.3 & 13.0 & 12.7 & 8.8 \\
\hline Other and losses & 83.3 & 91.7 & 94.6 & 96.1 & 72.6 \\
\hline
\end{tabular}

Sources: Statistics Department of the Ministry of Finance; and LUCELEC.

1/ Figures for Gasoline, Diesel, and Kerosene are for January - September, 2003. Figures for Electricity consumption are for January - August, 2003 
Table 24. St. Lucia: Merchandise Trade Volumes, Unit Values and Terms of Trade

\begin{tabular}{|c|c|c|c|c|c|}
\hline & 1999 & 2000 & 2001 & 2002 & $\begin{array}{r}\text { Est. } \\
2003\end{array}$ \\
\hline \multicolumn{6}{|c|}{$($ Index, $2000=100)$} \\
\hline \multicolumn{6}{|l|}{ Volumes } \\
\hline Exports & 90.0 & 100.0 & 91.2 & 91.6 & 98.3 \\
\hline Merchandise exports $1 /$ & 92.9 & 100.0 & 68.8 & 86.8 & 68.5 \\
\hline Tourism 2/ & 89.5 & 100.0 & 95.1 & 92.8 & 103.8 \\
\hline Merchandise imports 3/ & 99.3 & 100.0 & 89.9 & 88.7 & 112.4 \\
\hline \multicolumn{6}{|l|}{ Unit values } \\
\hline Exports & 108.0 & 100.0 & 93.0 & 89.3 & 91.8 \\
\hline Merchandise exports $1 /$ & 106.8 & 100.0 & 98.4 & 102.2 & 106.3 \\
\hline Tourism 4/ & 108.3 & 100.0 & 92.3 & 86.9 & 89.8 \\
\hline Merchandise imports 3/ & 100.5 & 100.0 & 96.8 & 99.4 & 100.4 \\
\hline Terms of trade & 107.5 & 100.0 & 96.1 & 89.8 & 91.4 \\
\hline (excluding tourism) & 106.2 & 100.0 & 101.6 & 102.8 & 105.9 \\
\hline \multicolumn{6}{|c|}{ (Annual percentage change) } \\
\hline \multicolumn{6}{|l|}{ Volumes } \\
\hline Exports & 0.9 & 11.2 & -8.8 & 0.5 & 7.3 \\
\hline Merchandise exports $1 /$ & -5.8 & 7.6 & -31.2 & 26.2 & -21.1 \\
\hline Tourism & 2.2 & 11.8 & -4.9 & -2.4 & 11.8 \\
\hline Merchandise imports 3/ & 13.9 & 0.7 & -10.1 & -1.3 & 26.7 \\
\hline \multicolumn{6}{|l|}{ Unit values } \\
\hline Exports & -0.4 & -7.5 & -7.0 & -4.0 & 2.7 \\
\hline Merchandise exports $1 /$ & -0.6 & -6.4 & -1.6 & 3.9 & 4.0 \\
\hline Tourism & -0.3 & -7.6 & -7.7 & -5.9 & 3.4 \\
\hline Merchandise imports 3/ & -7.2 & -0.5 & -3.2 & 2.7 & 1.0 \\
\hline Terms of trade & 7.3 & -7.0 & -3.9 & -6.5 & 1.7 \\
\hline (excluding tourism) & 7.1 & -5.9 & 1.6 & 1.2 & 3.0 \\
\hline
\end{tabular}

Source: Statistical Department, Ministry of Finance; and Fund staff estimates.

1/ Based on merchandise export items accounting for about 90 percent of total domestic exports

2/ Number of tourist arrivals.

3/ Based on data for main trading partners that account for at least 95 percent of imports.

4/ Average expenditure per day of each tourist 
Table 25. St. Lucia: Direction of Trade

(In percent of total)

\begin{tabular}{|c|c|c|c|c|c|}
\hline & 1998 & 1999 & 2000 & 2001 & 2002 \\
\hline Export destination & 100.0 & 100.0 & 100.0 & 100.0 & 100.0 \\
\hline United Kingdom & 65.1 & 63.5 & 55.9 & 40.6 & 37.6 \\
\hline United States & 13.8 & 12.1 & 14.8 & 19.0 & 20.0 \\
\hline CARICOM countries & 19.1 & 22.3 & 26.7 & 34.3 & 37.3 \\
\hline Barbados & 4.0 & 7.2 & 10.3 & 9.0 & 9.6 \\
\hline Guyana & 0.6 & 0.5 & 0.0 & 0.5 & 0.8 \\
\hline Jamaica & 0.0 & 0.0 & 0.0 & 0.3 & 0.5 \\
\hline Trinidad and Tobago & 5.7 & 3.8 & 1.8 & 11.4 & 11.7 \\
\hline OECS & 8.3 & 10.2 & 13.2 & 12.4 & 13.9 \\
\hline Other & 0.6 & 0.6 & 1.5 & 0.7 & 0.8 \\
\hline France & 0.1 & 0.1 & 0.0 & 0.2 & 0.1 \\
\hline Italy & 0.0 & 0.0 & 0.0 & 0.2 & 0.0 \\
\hline Rest of world & 2.0 & 1.9 & 2.6 & 5.6 & 4.87 \\
\hline Origin of Imports & 100.0 & 100.0 & 100.0 & 100.0 & 100.0 \\
\hline United Kingdom & 9.3 & 10.5 & 8.5 & 9.2 & 8.7 \\
\hline Canada & 3.4 & 3.3 & 3.8 & 3.4 & 2.9 \\
\hline United States & 36.6 & 39.6 & 37.5 & 38.3 & 39.3 \\
\hline CARICOM countries & 21.4 & 21.6 & 21.7 & 22.9 & 21.9 \\
\hline Barbados & 3.1 & 3.0 & 2.8 & 3.3 & 2.6 \\
\hline Jamaica & 1.0 & 0.9 & 0.8 & 0.9 & 1.1 \\
\hline Trinidad and Tobago & 12.9 & 14.1 & 14.4 & 14.2 & 15.1 \\
\hline OECS & 3.6 & 2.7 & 2.6 & 2.6 & 2.3 \\
\hline Other & 0.8 & 0.9 & 1.1 & 1.8 & 0.8 \\
\hline Germany & 1.4 & 1.0 & 1.0 & 0.9 & 1.2 \\
\hline France & 2.0 & 1.4 & 2.0 & 1.7 & 2.0 \\
\hline Netherlands & 1.7 & 1.2 & 1.1 & 1.3 & 1.2 \\
\hline Italy & 0.7 & 0.8 & 1.0 & 0.8 & 0.8 \\
\hline Japan & 5.2 & 5.6 & 4.5 & 4.8 & 3.4 \\
\hline Rest of world & 18.3 & 15.0 & 18.8 & 16.5 & 18.7 \\
\hline
\end{tabular}

Sources: Statistics Department of the Ministry of Finance. 
Table 26. St. Lucia: Selected Tourism Statistics

\begin{tabular}{|c|c|c|c|c|c|}
\hline & 1999 & 2000 & 2001 & 2002 & $\begin{array}{l}\text { Prel. } \\
2003\end{array}$ \\
\hline Total visitor arrivals & 622,059 & 726,254 & 747,181 & 648,355 & 628,849 \\
\hline Stay-over arrivals & 260,583 & 269,850 & 250,132 & 253,463 & 281,344 \\
\hline Hotel tourist & 221,496 & 229,373 & 212,612 & 215,444 & 239,142 \\
\hline Hotel business & 15,635 & 16,191 & 15,008 & 15,208 & 16,881 \\
\hline Private residence & 23,452 & 24,287 & 22,512 & 22,812 & 25,321 \\
\hline Excursionist & 10,243 & 12,853 & 7,137 & 7,712 & 6,787 \\
\hline Cruiseship & 351,233 & 443,551 & 489,912 & 387,180 & 340,718 \\
\hline Average daily expenditure (EC\$) & 272.1 & 251.3 & 232.0 & 218.3 & 225.7 \\
\hline Stay-over arrivals & 295.6 & 275.4 & 250.6 & 234.6 & 238.9 \\
\hline Hotel tourist & 318.1 & 287.9 & 260.2 & 242.4 & 249.8 \\
\hline Hotel business & 318.1 & 287.9 & 260.2 & 242.4 & 249.8 \\
\hline Private residence & 160.0 & 166.0 & 166.0 & 166.0 & 171.0 \\
\hline Excursionist & 140.4 & 121.5 & 121.5 & 121.5 & 122.7 \\
\hline Cruiseship & 116.1 & 113.0 & 141.3 & 116.7 & 117.9 \\
\hline Average length of stay (days) & 4.4 & 4.2 & 3.9 & 4.4 & 5.1 \\
\hline Stay-over arrivals & 9.2 & 9.7 & 9.7 & 9.7 & 10.1 \\
\hline Hotel tourist & 9.0 & 10.0 & 10.0 & 10.0 & 10.0 \\
\hline Hotel business & 3.5 & 3.5 & 3.5 & 3.5 & 3.5 \\
\hline Private residence & 14.5 & 11.0 & 11.0 & 11.0 & 15.5 \\
\hline Excursionist & 1.0 & 1.0 & 1.0 & 1.0 & 1.0 \\
\hline Cruiseship & 1.0 & 1.0 & 1.0 & 1.0 & 1.0 \\
\hline Visitor expenditure (millions of EC\$) & 748.2 & 772.6 & 678.2 & 623.0 & 720.1 \\
\hline Stay-over arrivals & 706.0 & 720.9 & 608.1 & 576.9 & 679.1 \\
\hline Hotel tourist & 634.2 & 660.3 & 553.3 & 522.3 & 597.3 \\
\hline Hotel business & 17.4 & 16.3 & 13.7 & 12.9 & 14.8 \\
\hline Private residence & 54.4 & 44.3 & 41.1 & 41.6 & 67.1 \\
\hline Excursionist & 1.4 & 1.6 & 0.9 & 0.9 & 0.8 \\
\hline Cruiseship & 40.8 & 50.1 & 69.2 & 45.2 & 40.2 \\
\hline \multicolumn{6}{|l|}{ Total visitor expenditure } \\
\hline In millions of U.S. dollars & 277.1 & 286.1 & 251.2 & 230.7 & 266.7 \\
\hline In percent of GDP & 41.4 & 41.9 & 38.4 & 34.1 & 38.5 \\
\hline \multicolumn{6}{|c|}{ (In percent change) } \\
\hline Total visitor arrivals & -1.3 & 16.8 & 2.9 & -13.2 & -3.0 \\
\hline Stay-over arrivals & 3.3 & 3.6 & -7.3 & 1.3 & 11.0 \\
\hline
\end{tabular}

Sources: Eastern Caribbean Central Bank; and Fund staff estimates. 
Table 27. St. Lucia: Stayover Tourist Arrivals by Country of Origin

\begin{tabular}{|c|c|c|c|c|c|}
\hline & 1999 & 2000 & 2001 & 2002 & $\begin{array}{r}\text { Est. } \\
2003 \\
\end{array}$ \\
\hline \multicolumn{6}{|c|}{ (Number of arrivals in thousands) } \\
\hline Total stayover arrivals & 260.6 & 269.9 & 250.1 & 253.5 & 281.3 \\
\hline United States & 83.5 & 97.5 & 91.2 & 94.0 & 98.5 \\
\hline United Kingdom & 69.9 & 73.4 & 66.5 & 63.3 & 73.9 \\
\hline Canada & 13.2 & 15.0 & 12.3 & 12.9 & 13.1 \\
\hline Caribbean & 60.3 & 54.6 & 60.2 & 66.4 & 70.9 \\
\hline France & 13.6 & 11.0 & 5.1 & 3.4 & 6.8 \\
\hline Germany & 8.7 & 7.3 & 4.8 & 3.9 & 4.5 \\
\hline Other & 11.5 & 11.0 & 10.0 & 9.5 & 13.6 \\
\hline \multicolumn{6}{|c|}{ (In percent of total) } \\
\hline Total arrivals & 102.8 & 106.5 & 98.7 & 100.0 & 111.0 \\
\hline United States & 32.9 & 38.5 & 36.0 & 37.1 & 38.9 \\
\hline United Kingdom & 27.6 & 29.0 & 26.2 & 25.0 & 29.1 \\
\hline Canada & 5.2 & 5.9 & 4.8 & 5.1 & 5.2 \\
\hline Caribbean & 23.8 & 21.5 & 23.7 & 26.2 & 28.0 \\
\hline France & 5.4 & 4.3 & 2.0 & 1.3 & 2.7 \\
\hline Germany & 3.4 & 2.9 & 1.9 & 1.6 & 1.8 \\
\hline Other & 4.5 & 4.4 & 4.0 & 3.7 & 5.4 \\
\hline
\end{tabular}

Sources: Statistics Department of the Ministry of Finance; Tourism Board; and Fund staff estimates. 
Table 28. St. Lucia: Summary External Public Debt

\begin{tabular}{|c|c|c|c|c|c|}
\hline & 1999 & 2000 & 2001 & 2002 & $\begin{array}{r}\text { Est. } \\
2003 \\
\end{array}$ \\
\hline \multicolumn{6}{|c|}{ (In millions of US dollars) } \\
\hline Outstanding debt at beginning of period & 154.8 & 170.0 & 197.1 & 214.4 & 259.8 \\
\hline Central government & 87.5 & 101.8 & 132.1 & 148.5 & 194.2 \\
\hline Bonded debt & 8.8 & 8.8 & 32.3 & 45.7 & 79.7 \\
\hline Loans & 78.7 & 93.0 & 99.8 & 102.8 & 114.6 \\
\hline Government-guaranteed & 62.2 & 63.2 & 61.7 & 56.4 & 53.7 \\
\hline Of which: Lucelec & 12.2 & 12.1 & 10.7 & 9.2 & 8.3 \\
\hline Other & 5.1 & 5.1 & 3.3 & 9.5 & 11.9 \\
\hline Total drawings & 23.3 & 41.5 & 46.0 & 65.3 & 84.9 \\
\hline Central government & 17.5 & 37.2 & 37.1 & 60.8 & 82.8 \\
\hline Bonded debt & 0.0 & 23.5 & 13.4 & 34.3 & 62.4 \\
\hline Loans & 17.5 & 13.6 & 23.7 & 26.5 & 20.4 \\
\hline Government-guaranteed & 5.2 & 4.3 & 1.5 & 1.1 & 2.1 \\
\hline Of which: Lucelec & 0.0 & 0.0 & 0.0 & 0.0 & 0.0 \\
\hline Other & 0.6 & 0.0 & 7.4 & 3.5 & 0.0 \\
\hline Debt service payments & 16.9 & 20.0 & 36.6 & 34.6 & 32.9 \\
\hline Amortization & 10.0 & 12.0 & 25.1 & 23.9 & 17.4 \\
\hline Central government & 3.7 & 5.2 & 19.1 & 17.9 & 12.5 \\
\hline Bonded debt & 0.0 & 0.0 & 0.0 & 0.4 & 6.0 \\
\hline Loans & 3.7 & 5.2 & 19.1 & 17.5 & 6.5 \\
\hline Government-guaranteed & 4.9 & 5.5 & 4.7 & 4.8 & 4.9 \\
\hline Of which: Lucelec & 1.0 & 1.4 & 1.4 & 1.0 & 0.8 \\
\hline Other & 1.4 & 1.3 & 1.2 & 1.2 & 0.0 \\
\hline Interest & 6.9 & 8.1 & 11.5 & 10.7 & 15.5 \\
\hline Central government & 3.5 & 4.7 & 8.1 & 7.9 & 13.3 \\
\hline Bonded debt & 0.7 & 2.1 & 3.4 & 4.1 & 8.5 \\
\hline Loans & 2.9 & 2.6 & 4.7 & 3.8 & 4.7 \\
\hline Government-guaranteed & 3.0 & 3.1 & 2.9 & 2.0 & 2.2 \\
\hline Of which: Lucelec & 0.5 & 0.5 & 0.4 & 0.3 & 0.3 \\
\hline Other & 0.4 & 0.3 & 0.5 & 0.7 & 0.0 \\
\hline Valuation adjustment & 1.9 & -2.4 & -3.6 & 4.0 & 2.3 \\
\hline Of which: Lucelec & 0.9 & 0.1 & -0.1 & 0.1 & -0.1 \\
\hline Outstanding debt end of period & 170.0 & 197.1 & 214.4 & 259.8 & 329.6 \\
\hline Central government & 101.8 & 132.1 & 148.5 & 194.2 & 267.0 \\
\hline Bonded debt & 8.8 & 32.3 & 45.7 & 79.7 & 136.0 \\
\hline Loans & 93.0 & 99.8 & 102.8 & 114.6 & 130.9 \\
\hline Government-guaranteed & 63.2 & 61.7 & 56.4 & 53.7 & 50.7 \\
\hline Of which: Lucelec & 12.1 & 10.7 & 9.2 & 8.3 & 7.3 \\
\hline Other & 5.1 & 3.3 & 9.5 & 11.9 & 12.0 \\
\hline \multicolumn{6}{|c|}{ (In Percent) } \\
\hline Total external debt (in percent of GDP) & 25.4 & 28.8 & 32.8 & 38.4 & 47.6 \\
\hline $\begin{array}{l}\text { Debt service (in percent of exports of goods } \\
\text { and nonfactor services) }\end{array}$ & 4.4 & 5.3 & 10.4 & 10.0 & 8.5 \\
\hline
\end{tabular}

Source: Ministry of Finance; and Fund staff estimates. 
Table 29. St. Lucia: Effective Exchange Rate Indices 1/

$$
(1990=100)
$$

\begin{tabular}{|c|c|c|c|}
\hline & $\begin{array}{c}\text { Real Effective } \\
\text { Exchange Rate 2/ }\end{array}$ & $\begin{array}{l}\text { Nominal Effective } \\
\text { Exchange Rate 2/ }\end{array}$ & $\begin{array}{c}\text { Relative Consumer } \\
\text { Prices } \\
\end{array}$ \\
\hline \multicolumn{4}{|l|}{1999} \\
\hline I & 111.2 & 128.7 & 86.4 \\
\hline II & 115.4 & 131.6 & 87.7 \\
\hline III & 116.8 & 131.7 & 88.7 \\
\hline IV & 118.7 & 130.9 & 90.7 \\
\hline \multicolumn{4}{|l|}{2000} \\
\hline I & 119.7 & 133.2 & 89.9 \\
\hline II & 122.3 & 136.6 & 89.5 \\
\hline III & 123.0 & 138.9 & 88.6 \\
\hline IV & 124.3 & 141.7 & 87.7 \\
\hline \multicolumn{4}{|l|}{2001} \\
\hline I & 122.0 & 139.8 & 87.2 \\
\hline II & 123.9 & 143.6 & 86.3 \\
\hline III & 122.5 & 142.6 & 85.9 \\
\hline IV & 123.1 & 142.7 & 86.3 \\
\hline \multicolumn{4}{|l|}{2002} \\
\hline I & 124.8 & 144.4 & 86.5 \\
\hline II & 122.4 & 141.2 & 86.7 \\
\hline III & 118.2 & 136.8 & 86.4 \\
\hline IV & 116.9 & 136.7 & 85.6 \\
\hline \multicolumn{4}{|l|}{2003} \\
\hline I & 112.2 & 132.5 & 84.8 \\
\hline II & 108.2 & 129.1 & 83.8 \\
\hline III & 107.4 & 129.3 & 83.0 \\
\hline IV 3/ & 103.5 & 125.9 & 82.2 \\
\hline 1999 & 115.5 & 130.7 & 88.4 \\
\hline 2000 & 122.3 & 137.6 & 88.9 \\
\hline 2001 & 122.9 & 142.2 & 86.4 \\
\hline 2002 & 120.6 & 139.8 & 86.3 \\
\hline 2003 3/ & 108.2 & 129.5 & 83.6 \\
\hline
\end{tabular}

Source: IMF Information Notice System.

$1 /$ Period averages.

$2 /$ Increases denote appreciation.

3/ Through November 2003. 


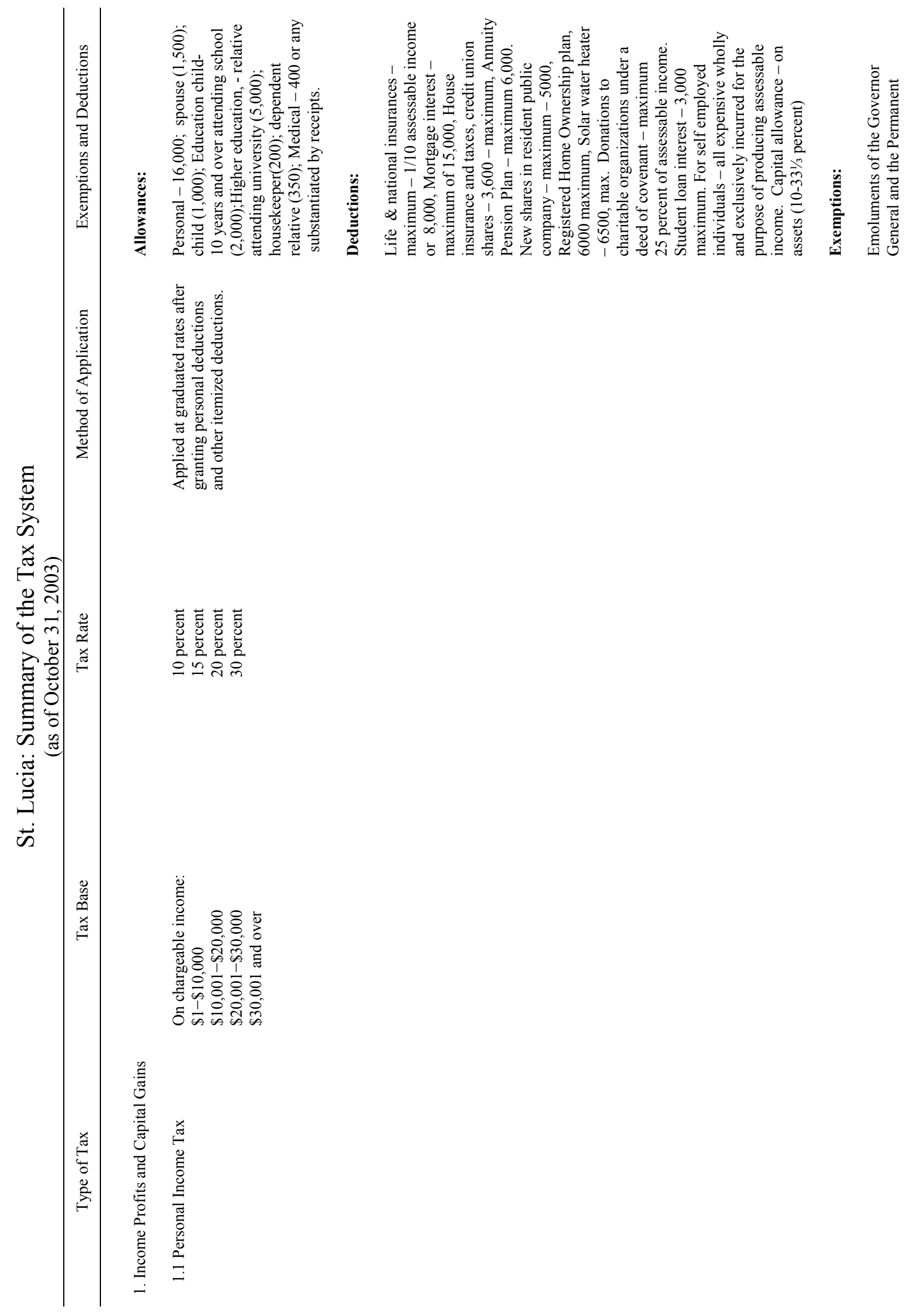




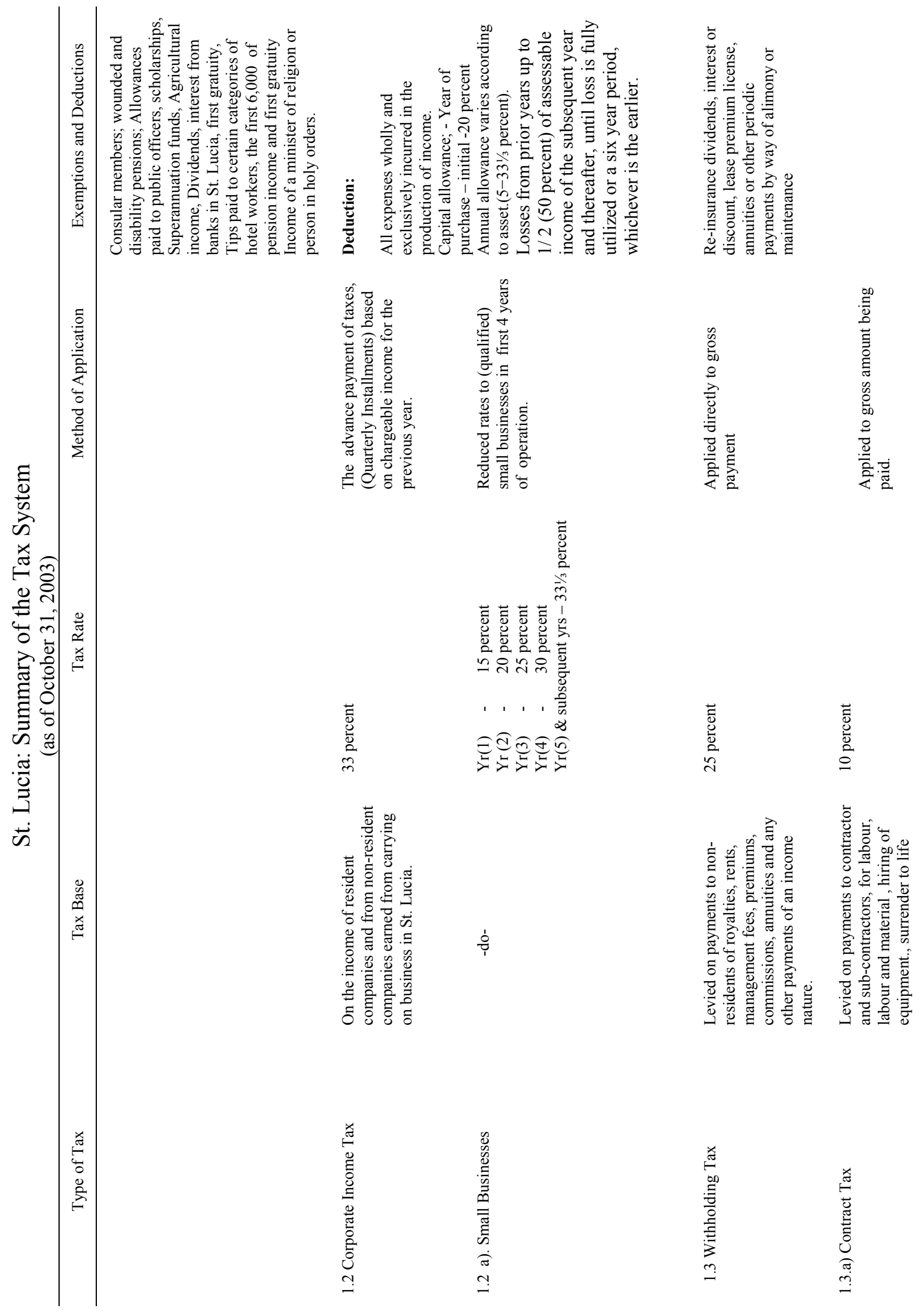




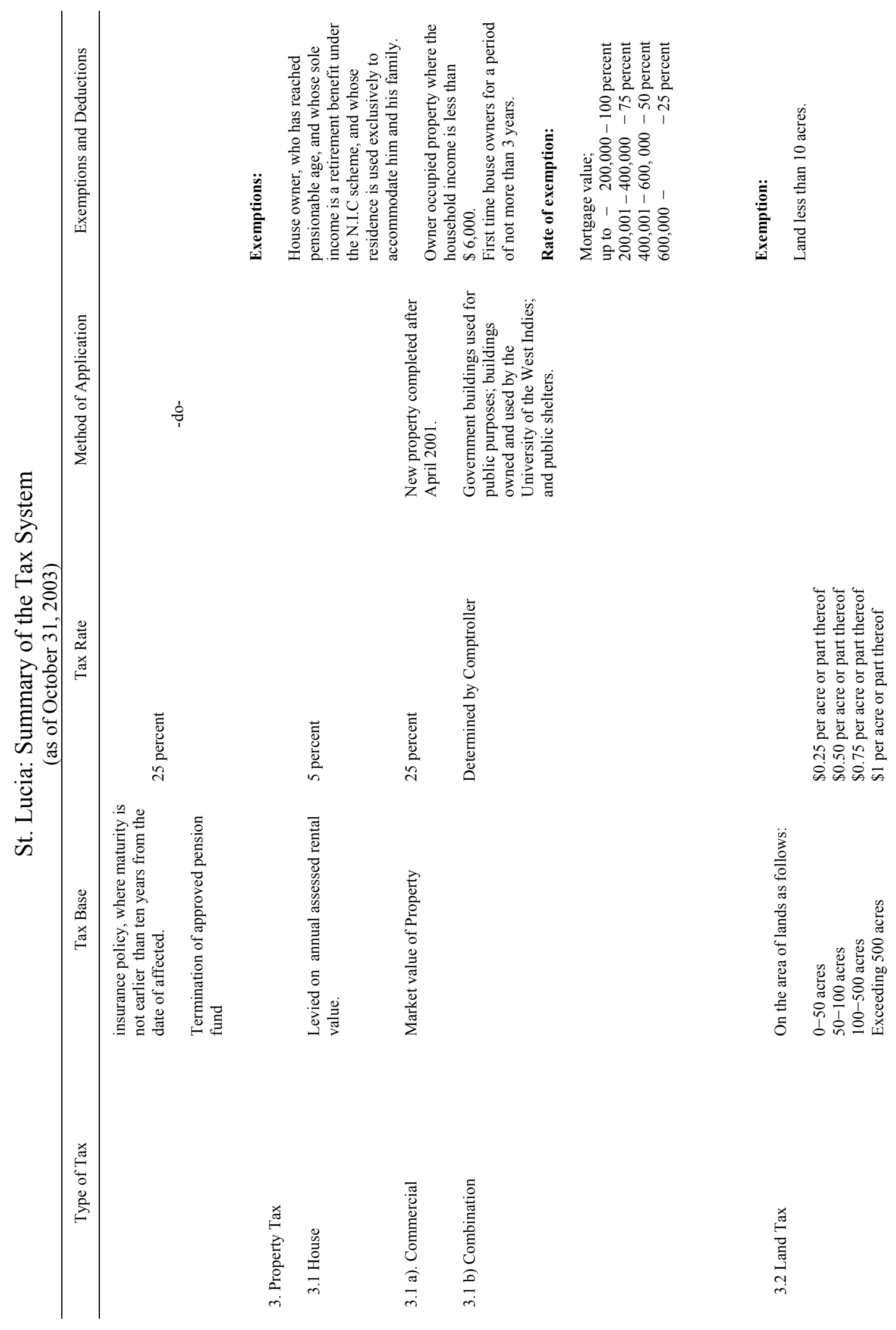




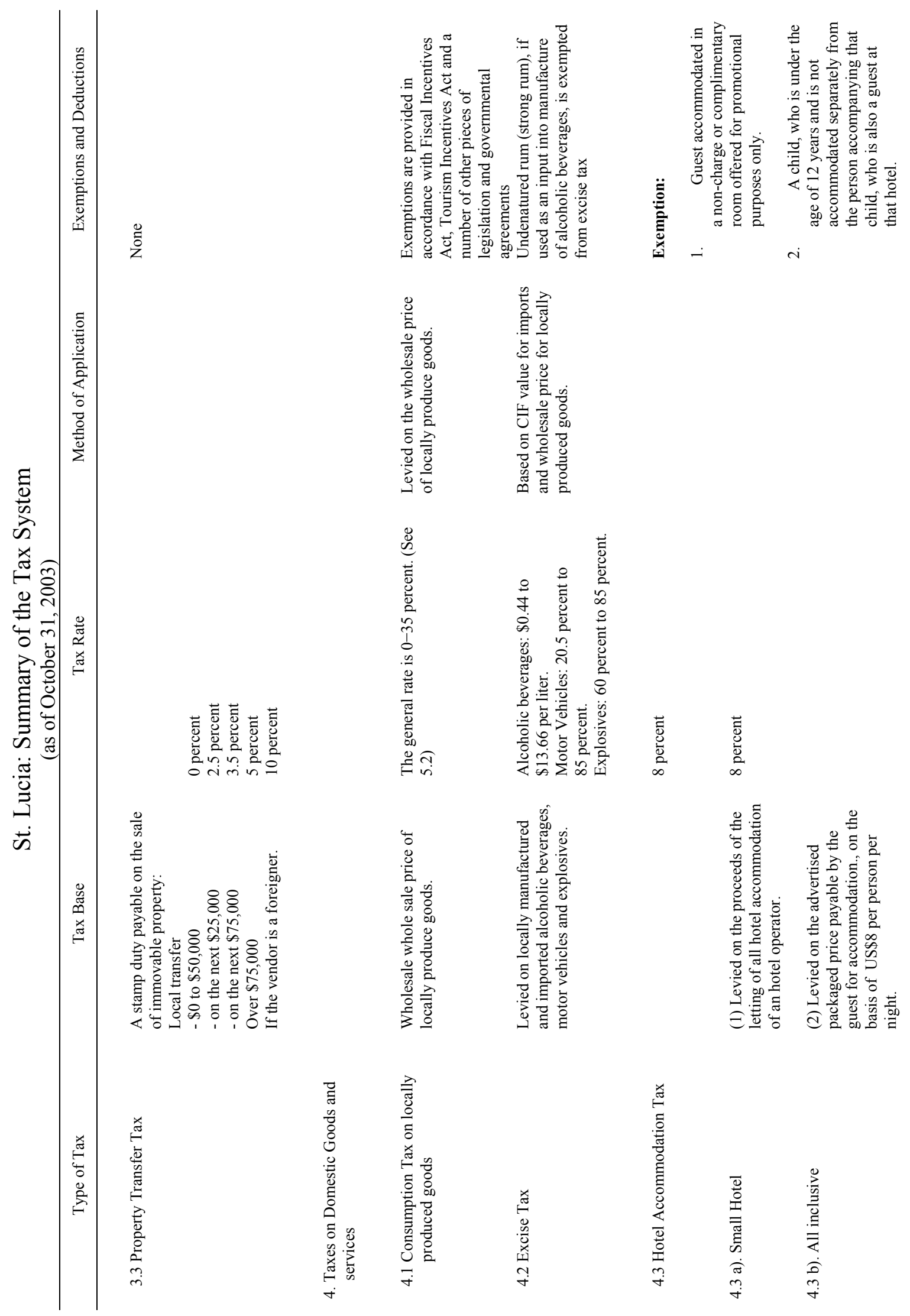




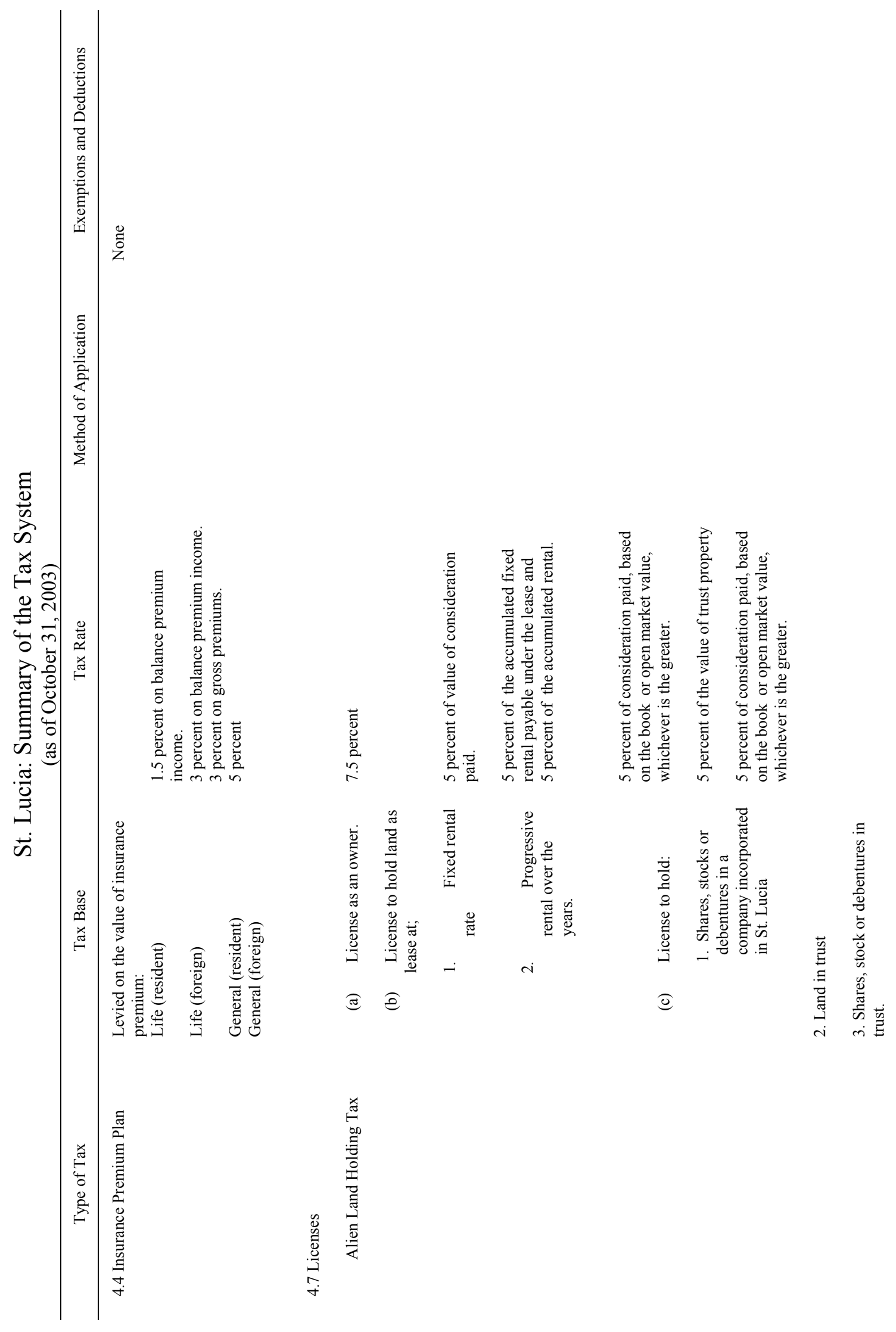




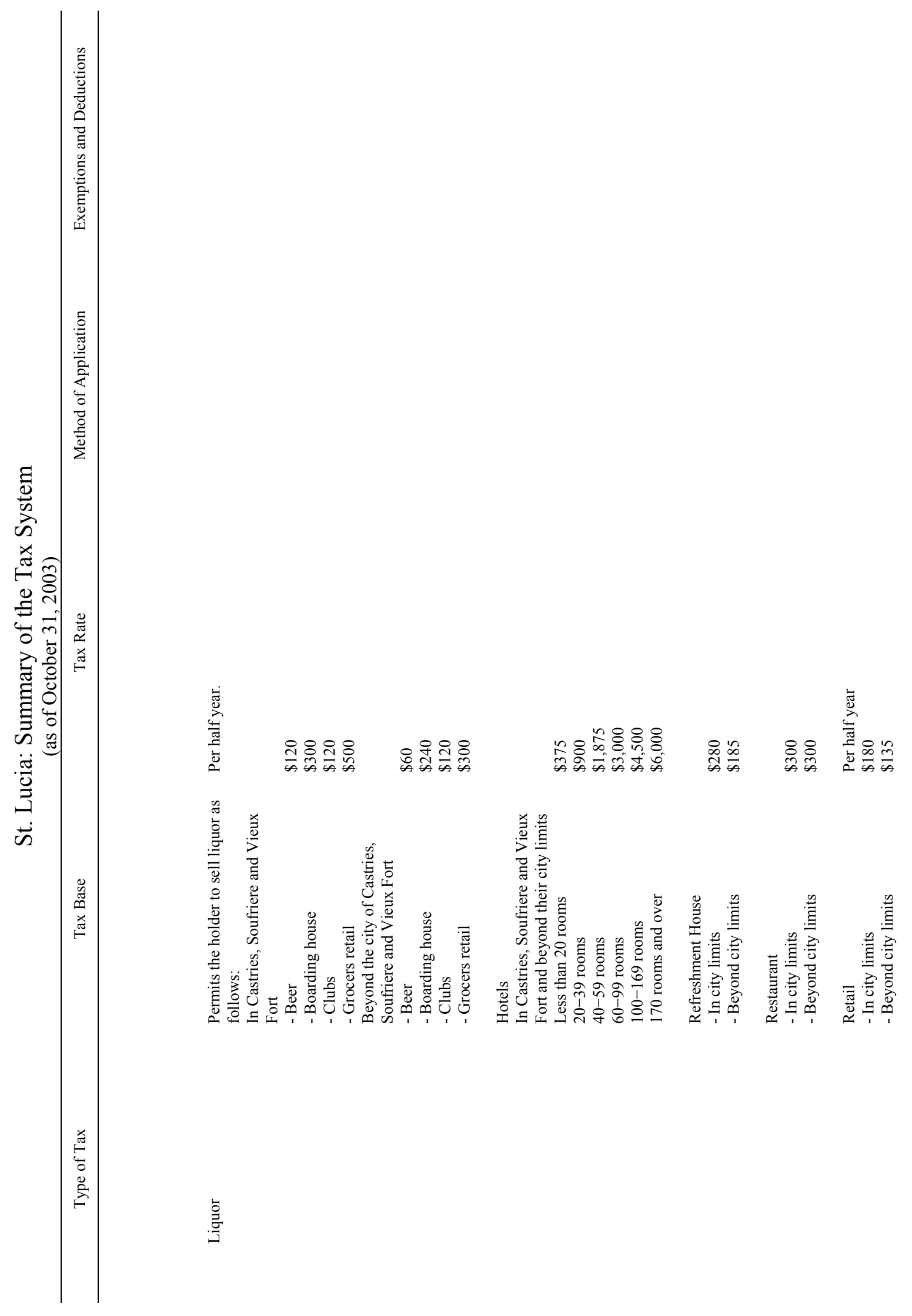




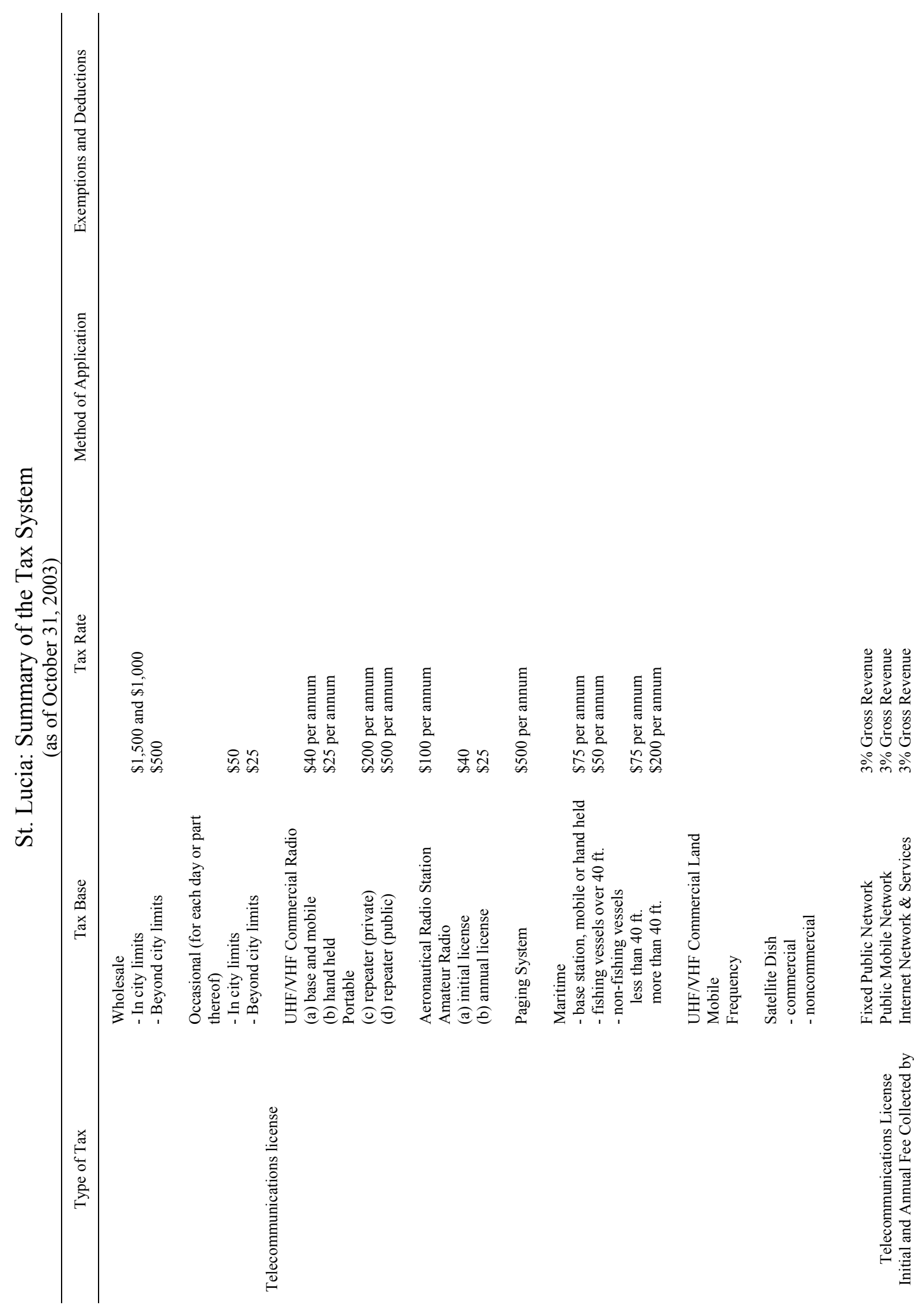


- 41 -

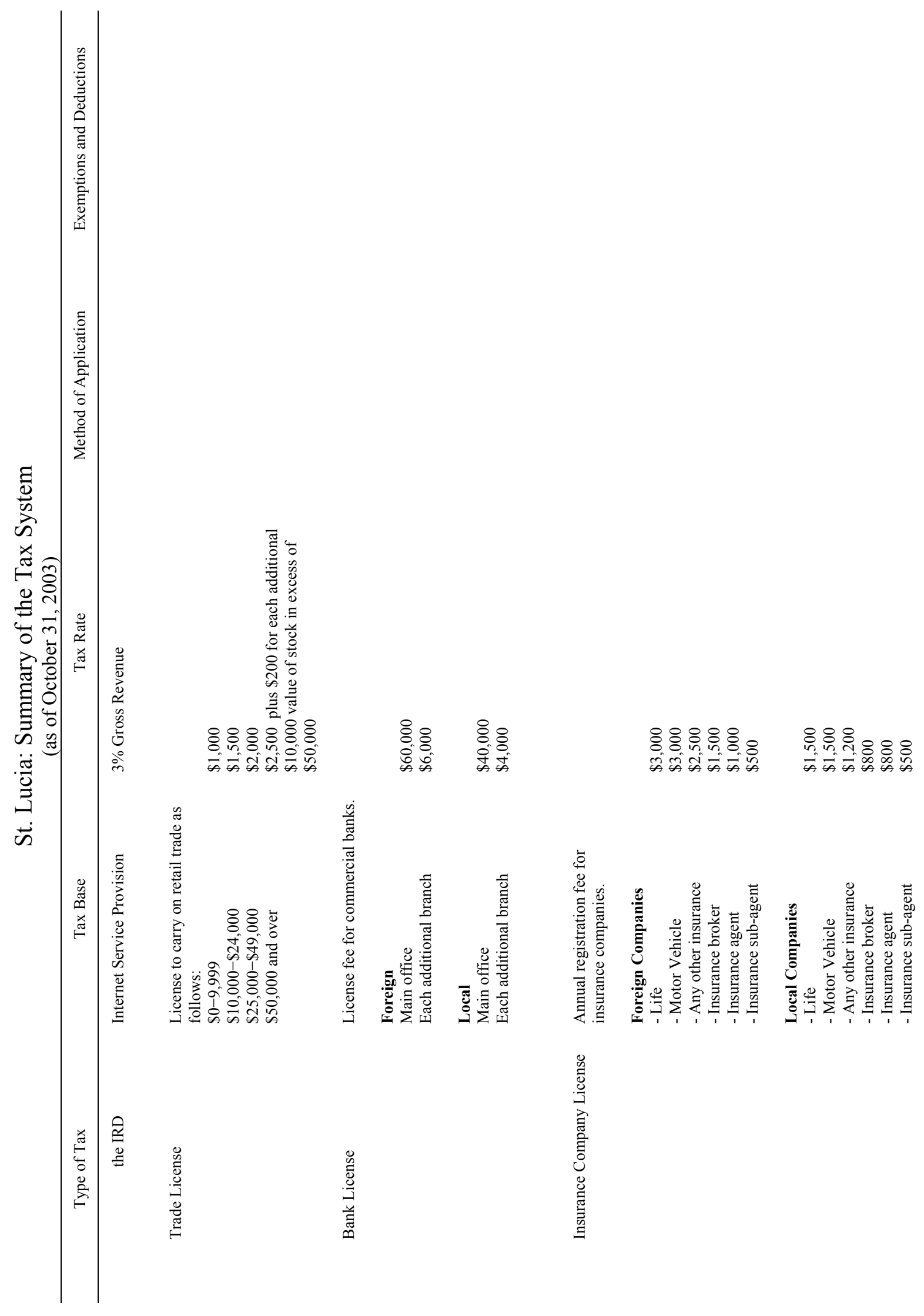




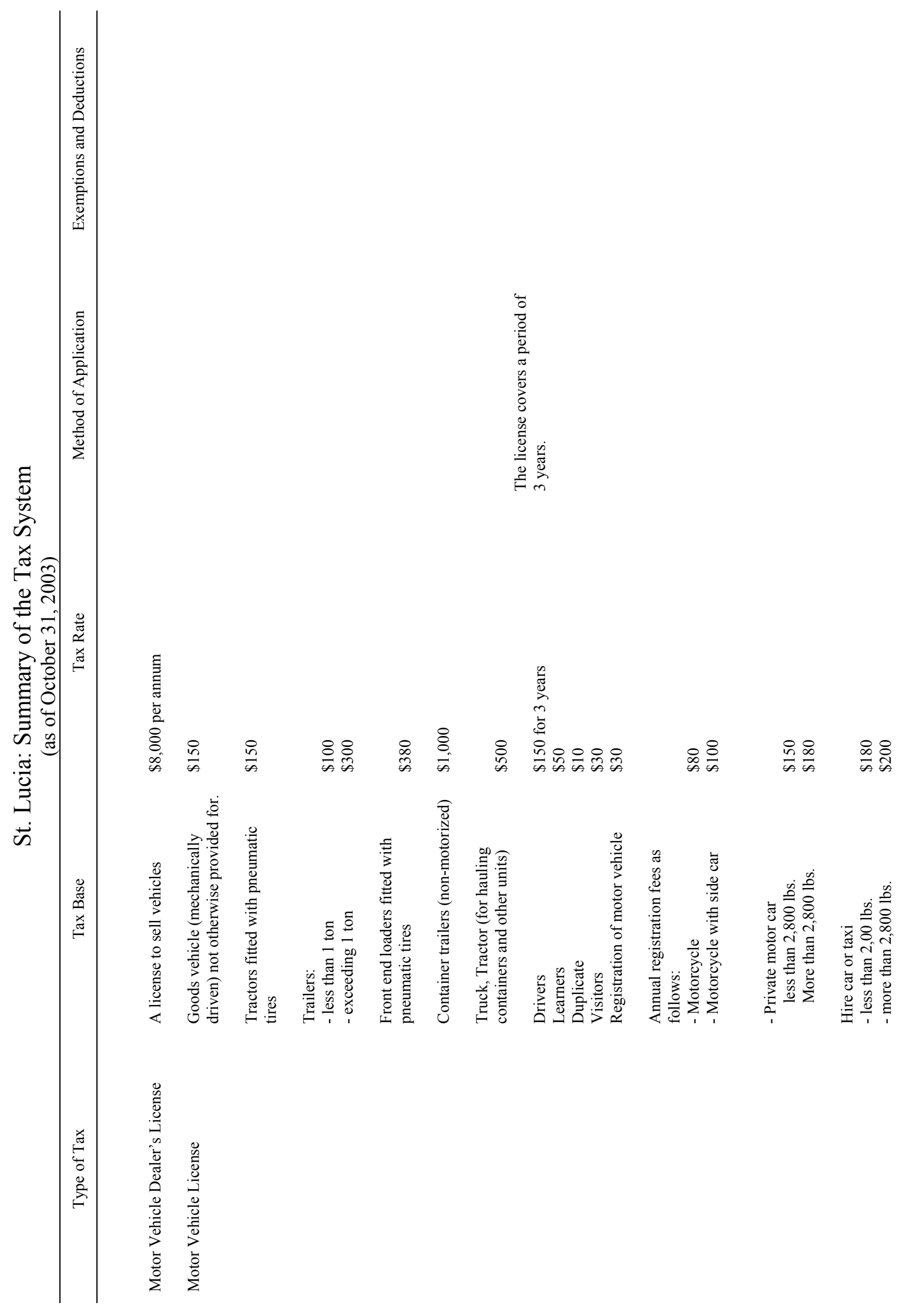




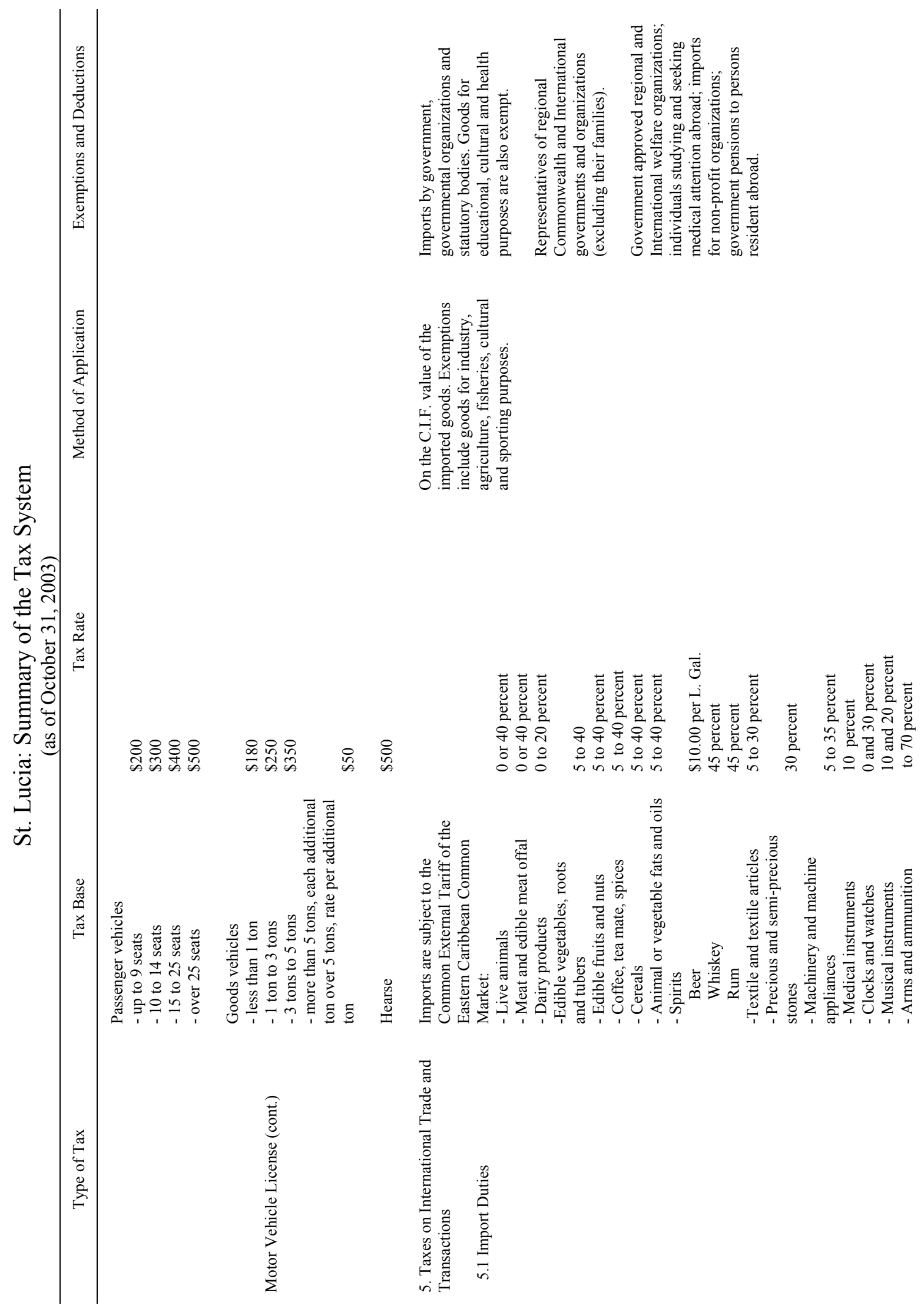




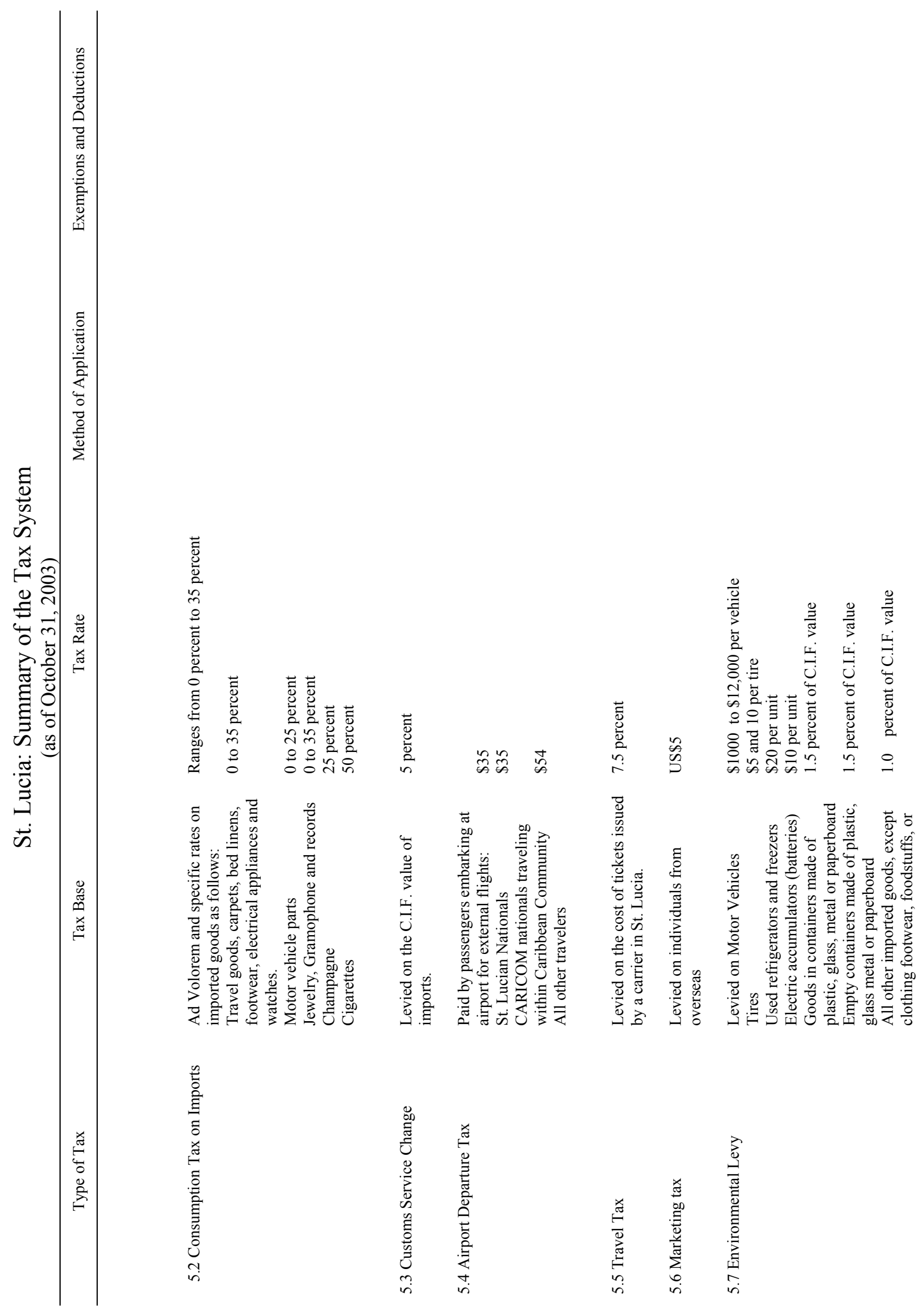




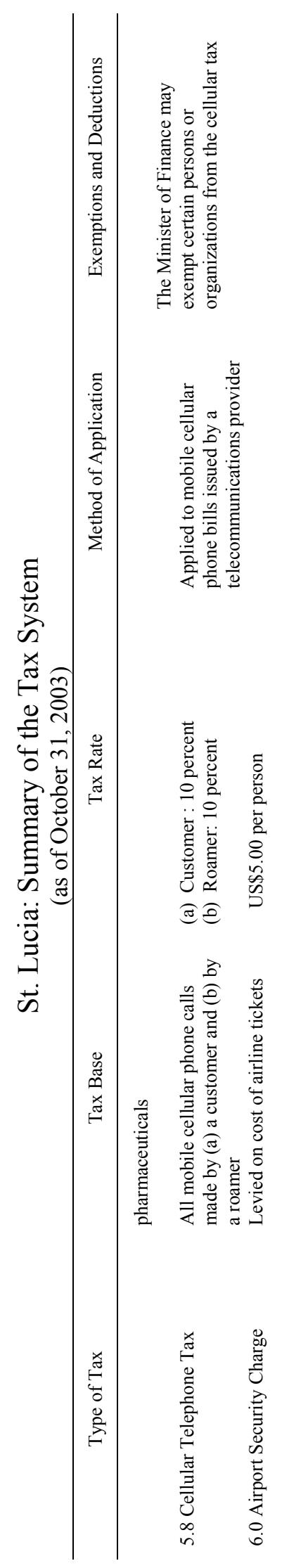

